\title{
A Clustering Solution for Analyzing Residential Water Consumption Patterns
}

\author{
Md Shamsur Rahim \\ Australian Artificial Intelligence Institute, School of Computer Science \\ University of Technology Sydney, Sydney, Australia \\ e-mail:mdshamsur.rahim@student.uts.edu.au \\ Khoi Anh Nguyen, Rodney Anthony Stewart \\ School of Engineering and Built Environment \\ Griffith University, Gold Coast, Australia \\ e-mail: k.nguyen@griffith.edu.au; r.stewart@griffith.edu.au \\ Tanvir Ahmed \\ Department of Computer Science \\ University of Central Florida, Orlando, Florida, United States of America \\ e-mail: tanvir.ahmed@ucf.edu \\ Damien Giurco \\ Institute for Sustainable Futures \\ University of Technology Sydney, Sydney, Australia \\ e-mail: damien.giurco@uts.edu.au \\ Michael Blumenstein* \\ Australian Artificial Intelligence Institute, Faculty of Engineering and IT \\ University of Technology Sydney, Sydney, Australia \\ e-mail: michael.blumenstein@uts.edu.au
}

\begin{abstract}
Water utility companies in urban areas face two major challenges: ensuring there is enough water for everyone during prolonged drought and maintaining adequate water pressure during the hours of peak demand. These issues can be overcome by applying data analytics and machine learning to the data gathered from digital water meters. For water conservation and demand management strategies to be effective, utility companies need to gain a better understanding of consumer behaviours, habits and routines. To accomplish this goal, we adapted a clustering approach to reveal residential water consumption patterns within metered data. In the experiment, we used two data sets (engineered features data set as well as the times of use and weighted probabilities of use data set) based on the data collected over 10 months from 306 households in Melbourne, Australia. For the engineered features data set, first, we identified the number of optimal clusters. We then performed extensive experiments to find the best clustering approach in terms of performance evaluation and clustering quality. We chose the hierarchical agglomerative clustering technique based on the nature of the data and the objective of the study. We observed that for the engineered features data set, k-means is the best performing clustering technique after considering performance metrics. For the other data set, we found that the number of clusters varies based on the type of water-consumption event, type of day (i.e., weekday or weekend), profiling interval and probability of use. In addition, we observed that insight into tap-water usage could be used to determine the population's adaptation of hygiene practices in an unprecedented time, such as the COVID-19 pandemic. Finally, we recommend that future clustering studies also employ aligned socio-demographic data and other key features.
\end{abstract}

Keywords: digital water meters; residential water consumption; clustering; customer segmentation; k-means clustering; hierarchical agglomerative clustering; consumption patterns; data analytics; machine learning.

\section{Introduction}

Water covers just over 70 per cent of the earth's surface. Still, a recent report identified water scarcity as one of the most significant global risks [1]. This is because water scarcity refers to the scarcity of potable water, which is fresh, safe, and easily accessible. Potable water accounts for only $0.014 \%$ of all water on earth [2]. In addition,

${ }^{*}$ Corresponding author 
unlike other natural resources, there is no alternative to water, and we cannot live without it. Therefore, any shortage of this resource can interrupt our lives completely. As an example of a community that suffered from a catastrophic drought, in 2017, South African officials coined the term Day Zero to represent the exact time in the future when municipal water would be turned off in Cape Town due to very low reservoir levels [3]. Fortunately, Cape Town escaped this fate by adopting dramatic water conservation, from $235 \mathrm{~L}$ per person per day (L/pp/dd) to $50(\mathrm{~L} / \mathrm{pp} / \mathrm{dd})$.

As in Cape Town, ensuring there is enough water for everyone during prolonged drought is one of the major challenges for water utility companies in urban areas. Managing demand during peak hours is another challenge for the urban utilities [4]. Digital water meters (DWMs) have been identified as a potential solution to water scarcity challenge due to their contribution of data to water conservation and peak demand management $[5,6]$. Effective water management strategies require an understanding of the behaviour of consumers and their habits and daily routines. This can be achieved by applying data analytics and machine learning techniques to DWM data [7]. Clustering is among the many data analytics and machine learning techniques that can be used to obtain a better understanding of water consumers' behaviour. Clustering is an unsupervised learning task where the items or objects are grouped based on some inherent similarity among them [8]. After performing clustering, similar objects are assigned in the same group and dissimilar objects are divided into different groups [9]. As clustering helps to identify previously unseen groups from the data, therefore, this technique has gained popularity in different areas such as health, biology, education, business, finance, energy, and water.

Several studies have been performed using clustering based on water-consumption data generated from DWMs. These previous studies can be divided into three categories: (i) customer segmentation, (ii) habit detection and profiling, and (iii) demand profiling. The aim of customer segmentation studies is to understand the waterconsumption behaviours of consumers by forming distinct groups of consumers based on their behaviours. The studies under this category are Vieira et al. [10], Garcia et al. [11] and Ji et al. [12], who found 5, 10 and 10 clusters, respectively. Among these studies, only Vieira et al. [10] performed clustering based on disaggregated water-consumption data and features derived from these data. The studies in the category of habit detection and profiling performed clustering based on the identification of habits (i.e., recurring behaviour) and consumption profiles; notable studies are [13-16]. However, none of these studies considered performing clustering based on changes in consumption over time, disaggregated data, type of day (i.e., weekday or weekend) or derived features. Finally, in the demand profiling studies, water consumers are categorised based on their level of water demand [7, 17]. Only Cominola et al. [7] considered disaggregated water consumption for clustering. We have observed various limitations in the existing studies, such as not considering disaggregated consumption data or recent changes in behaviour and that the derived features or patterns are not based on the type of day. However, to fully utilise the data collected from DWMs, these research gaps should be explored. In addition, recent studies [18, 19] have identified the potential of a personalised recommender system for promoting water-conscious behaviour and proposed an advanced customer-profiling approach [20,21]. Therefore, a suitable clustering study is required that can be used to implement a personalised recommender system for water conservation and demand management.

The primary motivation behind this study is to overcome the limitations of the existing clustering studies by introducing a clustering solution that would provide a better understanding of households' water consumption patterns, and it would be suitable for a recommender system in order to promote water-conscious behaviour [18]. To accomplish this task, we have considered a new and advanced profiling approach that offers in-depth details on consumer water-consumption patterns. This profiling approach is based on disaggregated water-consumption data. It accommodates changes in behaviour with time, separately considers the consumption patterns on weekdays and weekends and includes derived features [20]. Therefore, this approach helps to understand and track changes in behaviour at a household level and to make effective recommendations for promoting waterconscious behaviour. However, not all patterns in water consumption are unique, as they tend to be similar among consumers who belong to the same group. In this scenario, identifying the groups of consumers with similar consumption patterns can improve the performance of finding neighbours in collaborative filtering [22]. In addition, it can be useful for performing social comparisons, as social comparisons are effective in promoting water conservation [23]. Figure 1 shows a broad view of the personalised water-conscious recommender system. In addition, clustering the data gathered from this new profiling approach can reveal previously unseen waterconsumption patterns. Therefore, we have chosen to investigate the application of clustering techniques for the purpose of exploring water-consumption patterns based on advanced customer profiling. 


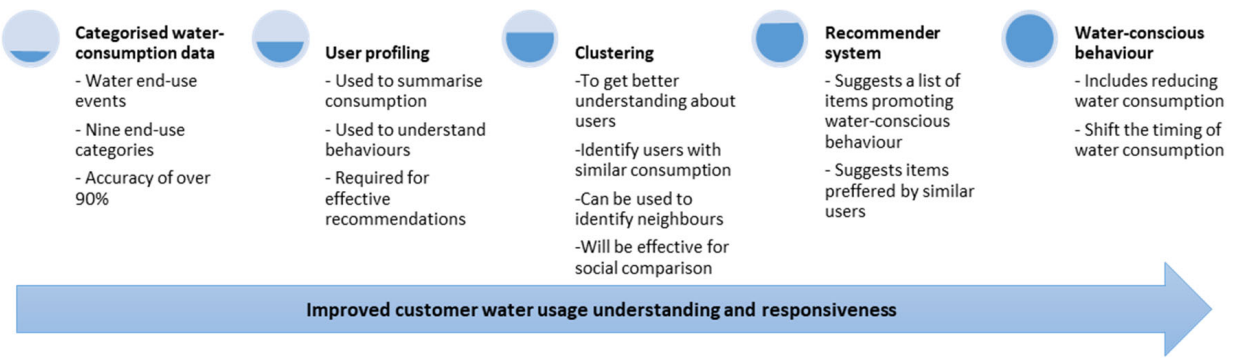

Figure 1. Broad view of the personalised water-conservation system.

In this study, we have performed clustering on two data sets: an engineered features (EF) data set and a data set including times of use and weighted probabilities of use (TUWPU) of water by consumers. The EF data set was created after performing feature engineering on residential water-consumption data. Based on the existing literature, statistical techniques were used to create 13 new features. Later, with the help of the elbow method [24], the optimal number of clusters was determined, and several clustering techniques were applied to identify the most suitable in terms of running time and clustering quality metrics. For the EF data set, five clusters were found to be optimal, and the characteristics of these clusters were investigated. We concluded that k-means would be the most suitable clustering technique after considering performance and clustering quality metrics. The TUWPU data set provided a weighted probability of an event happening at a particular time at three different intervals (i.e., 15 minutes, 30 minutes and 60 minutes) for five water end-use categories (shower, bathtub, washing machine, dishwasher, gardening) for different day types (i.e., weekday, weekend). In this scenario, we performed hierarchical agglomerative clustering as representing data items in a hierarchy is beneficial to data summarisation and visualisation [24]. Also, different linkage methods were employed to identify the best one, as clustering quality may vary depending on the linkage methods. After performing hierarchical agglomerative clustering, we observed that the optimal number of clusters varied based on the profiling interval, water-consumption event and type of day (i.e., weekday or weekend). However, among the three profiling intervals (i.e., 15 minutes, 30 minutes and 60 minutes), the 15-minute profiling interval outperformed the 30-minute and 60-minute profiling intervals to form clusters based on the similarity of consumer behaviour.

This study addresses many of the research gaps in the existing literature on clustering residential waterconsumption data and presents previously unseen and significant findings. The major contributions of this study are as follows:

- It has proposed a detailed clustering solution to effectively apply multiple clustering techniques to analyse water consumption data that overcomes the limitations of existing clustering studies.

- It has identified a suitable clustering technique in terms of run time, scalability and cluster quality metrics for the EF data set.

- It has identified the most suitable profiling interval and linkage method in terms of cluster quality metrics.

- It has observed previously unseen, new, insightful traits about the groups of households that can be used to design future water conservation and demand management programs.

- It has observed that insights from tap-water usage can be used to determine how hand-hygiene practices have adapted to preventing infection from viruses such as COVID-19.

\section{Related Work}

Several studies have been performed on the clustering of digital data on residential water metering, and they have had different goals. Based on their purpose, these studies can be divided into three categories: customer segmentation, habit detection and profiling and demand profiling. Though it is possible to perform segmentation or clustering based on other data sources such as water-billing data [25], for this study, we have considered any study that utilised data collected from DWMs. We have evaluated related work against four criteria: 1) the ability of the data set used in the study to accommodate recent changes in behaviour, 2) the nature of the consumption data (i.e., aggregated or disaggregated), 3) consideration of the difference in consumption patterns during weekdays and weekends and 4) the inclusion of derived features. 
The general aim of the studies we reviewed under the category of customer segmentation was to understand waterconsumption behaviours among consumers by forming distinct groups. To understand the overall efficiency of indoor water usage, clustering techniques were applied in a study [10] to compare these techniques and their efficiency patterns with those used by peers. In this study, the authors calculated the averages of the water-use device characteristics and derived an efficiency index. Later, they applied HAC using Ward's method based on the efficiency index, and they found five clusters. However, the data set used in their study was unable to consider recent changes in behaviour or changes in patterns based on the type of day (i.e., weekday or weekend). To understand the behaviour of residential water consumers and to predict water demand, Garcia et al. [11] applied the $k$-means clustering technique to DWM data and found 10 clusters. Similarly, in another study, fuzzy clustering was proposed for analysing urban residential water-consumption data collected at a 15 -minute interval [12]. They found 10 groups from 20 users after clustering residents by family structure, job type or lifestyle based on their water-consumption data.

On the other hand, the studies under the category of habit detection and profiling performed clustering by identifying habits (i.e., recurring behaviours) and consumption profiles. Based on the combination of k-means clustering and PCA on hourly consumption data, three profiles were identified by Cominola et al. [14] on the basis of eigenbehaviours (i.e., recurrent water-consumption behaviour). In contrast, clustering using signature patterns was proposed by Cardell-Oliver [15] to explain how households use water. The author found four signature patterns and recommended further research be performed to identify the best way to present the findings to maximise water conservation. Later, Wang et al. [26] proposed a novel algorithm for identifying all routines of variable lengths efficiently. The proposed algorithm expands longer candidate sub-sequences gradually instead of clustering sub-sequences repeatedly. In another study, to identify relevant water-usage profiles, Cheifetz et al. [13] applied a mixture of $k$-means and Fourier regression techniques on hourly consumption data. From residential consumption data, they found three clusters and observed two consumption peaks at 10:00 am and 8:00 pm.

Last, to predict and manage demand, studies on demand profiling categorised water consumers based on their level of demand. It is rare for clustering studies to consider disaggregated water consumption. However, in a recent study, disaggregated water-consumption data were used for customer segmentation. In this study, after extracting eigenbehaviour, dimension-reduction techniques were used: k-means clustering and t-distributed stochastic neighbour embedding. The authors found three main water end-use profile clusters. However, the study did not include derived features or consider recent changes in behaviour or different day types (i.e., weekday or weekend). In another study, various clustering methods (e.g., k-means clustering, dendrograms and selforganising maps) were applied to standardised monthly aggregated time series [17]. They found that a selforganising map with k-means clustering produces the best result with 19 clusters. Table 1 represents the summary of the related studies based on the evaluation criteria mentioned in this section.

Table 1. Summary of the relevant work against evaluating criteria

\begin{tabular}{|c|c|c|c|c|c|}
\hline Category & Study & $\begin{array}{l}\text { Considered } \\
\text { recent change? }\end{array}$ & $\begin{array}{l}\text { Based on } \\
\text { disaggregated data? }\end{array}$ & $\begin{array}{l}\text { Different type } \\
\text { of days? }\end{array}$ & $\begin{array}{l}\text { Considered } \\
\text { derived features? }\end{array}$ \\
\hline \multirow{3}{*}{$\begin{array}{l}\text { Customer } \\
\text { Segmentation }\end{array}$} & [10] & No & Yes & No & Yes \\
\hline & [11] & No & No & No & No \\
\hline & {$[12]$} & No & No & No & No \\
\hline \multirow{4}{*}{$\begin{array}{l}\text { Habit detection } \\
\text { and profiling }\end{array}$} & [13] & No & No & No & No \\
\hline & [14] & No & No & No & No \\
\hline & [15] & No & No & No & No \\
\hline & {$[16]$} & No & No & No & No \\
\hline \multirow{2}{*}{$\begin{array}{l}\text { Demand } \\
\text { profiling }\end{array}$} & [7] & No & Yes & No & No \\
\hline & {$[17]$} & No & No & No & No \\
\hline
\end{tabular}


Table 1 shows that most of these studies suffer from the limitations of not having considered recent changes in behaviour, derived features or patterns based on the type of day and of having considered only aggregated data. Though a small number of recent studies had addressed some of these limitations before the current study began, there remained a need to conduct a clustering study on residential DWM data to address these limitations. Using the EF data set and TUWPU data sets, this study has addressed these limitations.

\section{Methodology}

This study's methodology of performing a comprehensive investigation of residential waterconsumption patterns through clustering can be divided into four consecutive steps: 1) initial data collection and processing, 2) data set preparation, 3) experimentation, and 4) result analysis. In Step 1, raw data were collected through DWMs and categorised end-use data were acquired with the help of Autoflow [6, 27]. In Step 2, data sets for clustering were prepared by employing an advanced customer-profiling technique proposed by Rahim et al. [20, 21]. In Step 3, two clustering methods were applied to identify different groups of customers with similar consumption patterns. Finally, in Step 4, the output generated from the previous step was analysed. Figure 2 illustrates the four steps, and they are described in the following subsections.

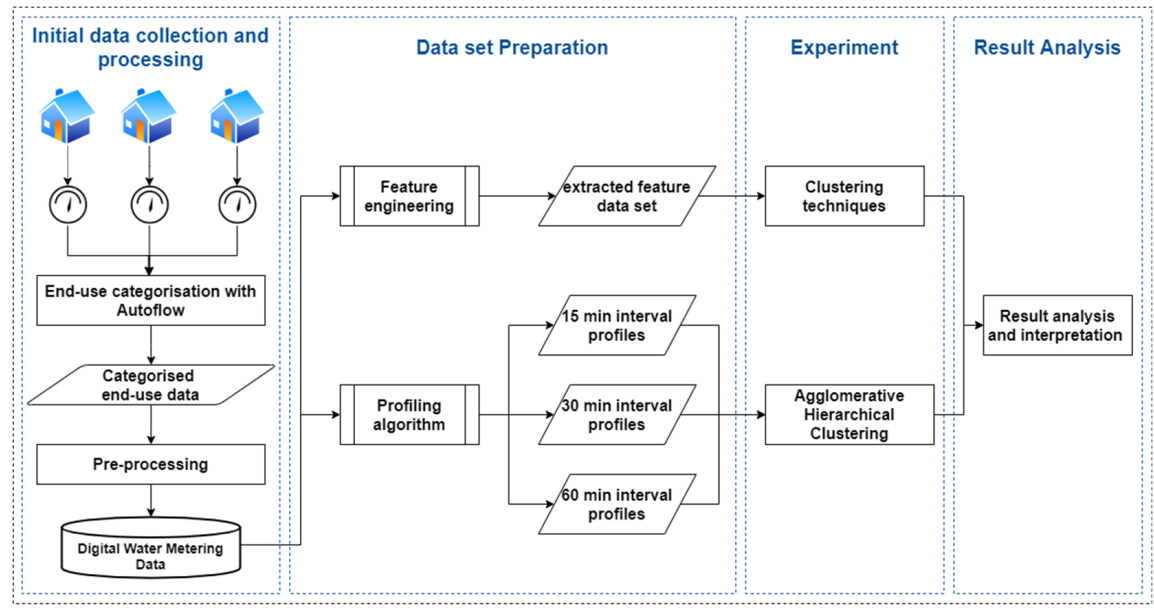

Figure 2. Steps in the methodology.

\subsection{Step 1: Initial data collection and preparation}

The initial data for this study originated from the high-resolution DWMs installed at 306 single standalone household units in various suburbs of Melbourne, Australia. The data set consisted of water-consumption data gathered at 5-s intervals over a period of 10 months (February-December 2010). The collected raw data were then analysed by Autoflow [27, 28], an intelligent metering system that outputs a categorised end-use data set. The categorised end-use data set consisted of nine end-use water categories: taps, dishwashers, leaks, evaporative coolers, clothes washers, showers, toilets, irrigation and bathtubs, with an accuracy of $90 \%$. This was the primary data set for this study.

After the primary data set was prepared, initial pre-processing tasks were performed. First, only the waterconsumption events that occurred between 6:00 am and 11:59 pm were considered. The remaining events that occurred between 12:00 am and 5:59 am were discarded due to the insignificant number of events and volume of water consumed during this period. Table 2 summarises the data set after selection for events that occurred between 6:00 am and 11:59 pm.

Table 2. Summary of data set after selecting events based on time of occurrence 


\begin{tabular}{ll}
\hline Item & Description \\
\hline \hline Number of households & 306 \\
Data collection duration & $2010-02-11$ to $2010-10-11$ \\
Hours considered & $6: 00 \mathrm{am}-11: 59 \mathrm{pm}$ \\
Data collection interval & 5 seconds \\
Number of end-use categories & 9 \\
Number of events & $56,310,576$ \\
Total shower events & 181,358 \\
Total bathtub events & 18,274 \\
Total clothes washer events & 30,996 \\
Total dishwasher events & 34,524 \\
Total irrigation events & 9,975 \\
Total toilet events & $1,173,902$ \\
Total tap events & $4,152,386$ \\
Total leak events & $50,709,161$ \\
\hline
\end{tabular}

Next, two end-use categories, evaporative coolers and leaks, were excluded from the data set. Evaporative coolers were excluded because they consume less than one per cent of the total water consumed [29]. Leaks were excluded because a leak is a continuous event, and one long continuous event represents a combination of many small, discrete events recorded at the interval of 5 seconds [20]. Therefore, to reduce computational time and improve scalability, all the leak events were omitted. In the last step of pre-processing, the type of day (weekend or weekday) was extracted from the given date, as water-consumption patterns vary based on this factor [20]. Table 3 describes the data set after this pre-processing step.

Table 3. Description of the attributes in the final dataset

\begin{tabular}{lll}
\hline Attribute & Description & Example \\
\hline \hline Site & Unique identifier for each household in the dataset & Site001, Site002 \\
Start date & Start date of an event & $11-$ Feb-2010 \\
Start time & Start time of an event & $08: 38: 15$ \\
End date & End date of an event & $11-$ Feb-2010 \\
End time & End time of an event & $08: 42: 10$ \\
Category & Water end-use category & Shower, dishwasher, etc. \\
Duration & Event duration time & $0: 03: 55$ \\
Volume & Quantity (L) of water used in an event & 36.81 \\
Max flow & Maximum flow rate (L/min) recorded for an event & 10.83 \\
Mode flow & Mode of flow rate (L/min) recorded for an event & 9.33 \\
Cyclic event & Multiple intakes of water during one single event & C1 (Cycle 1 for washing \\
& & machine), D5 (Cycle 5 for \\
Type of day & A weekend is Saturday or Sunday; a weekday is Monday to Friday & Weekend, weekday \\
& (inclusive) & \\
\hline
\end{tabular}

\subsection{Step 2: Data set preparation}

To accomplish the objectives of this study, two final data sets were derived from the primary data set: (i) the TUWPU data set and (ii) EF data set. The TUWPU data set provides the weighted probability that an event will occur at a particular time at three different intervals ( 15 minutes, 30 minutes and 60 minutes). In contrast, the EF data set consists of new predictor variables created using feature engineering. The following subsections describe the preparation of these data sets in detail.

\subsubsection{Time of use and weighted probability of use data set}

The TUWPU data set was prepared based on the profile-creation algorithm proposed by Rahim et al. [20]. This algorithm provides profiling at three different intervals: 15 minutes, 30 minutes and 60 minutes. For each profiling interval, a probability distribution (PD) was computed across three time periods for each household, for 
each event across different times and for each type of day. Later, these three PDs were combined after they had each been assigned a different weight. Algorithm 1 represents the steps in profile creation.

Here,

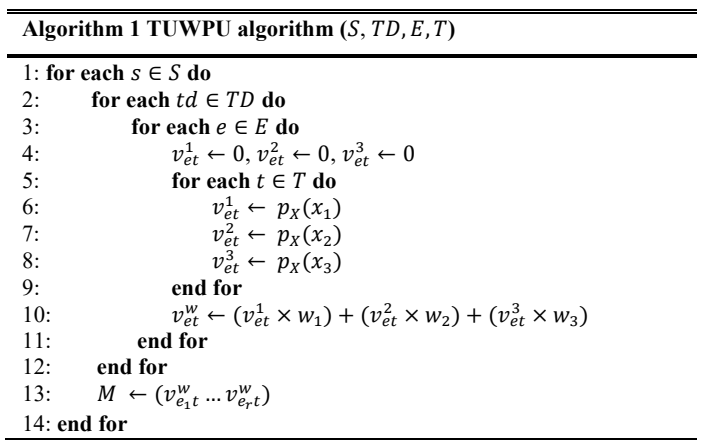

$S$ is the set of households, where $S=\{$ Site 001, Site $002, \ldots$, Site 307$\}$,

$T D$ is the set of types of day, where $T D=\{$ weekday, weekend $\}$,

$E$ is the set of events, where $E=\{$ shower, bathtub, clothes washer, dishwasher, irrigation $\}$,

$v_{e t}$ is an n-dimensional vector,

$T$ is the set of times from 6:00 am to $11: 59 \mathrm{pm}$ at a 15-minute, 30-minute or 60-minute interval,

$p_{X}(x)$ is a probability mass function,

$v_{\text {etw }}$ is a weighted n-dimensional vector,

$w$ is the weight assigned to PD vectors and

$M$ is the matrix by concatenating all vectors.

At Step 4, three $n$-dimensional vectors $v_{e t}^{1}, v_{e t}^{2}$, and $v_{e t}^{3}$ are initialised to 0 to calculate and store the probability distribution of event $e$ over a given time period for household $s$, where each $v_{e t}=\left(v_{e t_{1}}, v_{e t_{2}}, \ldots, v_{e t_{n-1}}, v_{e t_{n}}\right)$. Here, $v_{e t_{1}}, v_{e t_{2}}, \ldots, v_{e t_{n-1}}, v_{e t_{n}}$ are the components of vector $v_{e t}$, where each of the component represents the probability of occurring an event e for the given time t. In addition, the number of dimensions depends on the value of the profiling interval. For example, when profiling at the 30 -minute interval, there are 35 dimensions, as there will be 35 intervals of 30 minutes in this time period (i.e., $6: 00 \mathrm{am}, 6: 30 \mathrm{am}, 7: 00 \mathrm{am}, \ldots, 11: 30 \mathrm{pm}$ ). The reason for taking these three vectors is to capture the probability distribution in three different time periods (i.e., latest 18 days, previous 18 days and all previous days). In this way, the first PD vector $v_{e t}^{1}$ represents the distribution for the most recent days (i.e., the latest 18 days), the second PD vector $v_{e t}^{2}$ represents the previous 18 days, and the last PD vector $v_{e t}^{3}$ denotes the PD for all previous historical data. Next, in Step 6-8, a probability mass function (PMF) is used to understand the behaviour or habits of the users. PMF is a function that provides the probability that a discrete random variable is exactly equal to some value. Formally this function $p: \boldsymbol{R} \rightarrow[0,1]$ can be defined using equation 1 .

$$
p_{X}\left(x_{i}\right)=P\left(X=x_{i}\right) \text { for }-\infty<x<\infty
$$

Equation 1 provides the probability distribution (PD) for each event $e$ across time $t$ for the given type of day $t d$ and assign it to $v_{e t}$. Equation 1 can be further expanded to equation 2 .

$$
P\left(X=x_{i}\right)=\frac{\left|s t d e_{i} t\right|}{\left|s t d e_{i}\right|}
$$

Where $\left|s t d e_{i} t\right|$ provides the number of occurrences of an event $e_{i}$ at a particular time $t$, for household $s$, and for the type of day $t d ;\left|s t d e_{i}\right|$ provides the total of occurrences of an event $e_{i}$ for household $s$, and for the type of day $t d$.

Later, in Steps 10, the most recent data were given higher weights as they captured better changes in recent behaviour. Therefore, after assigning a higher weight to the first PD vector and lower weights to the other PD vectors, that is $w_{1}$ to $v_{e t}^{1}, w_{2}$ to $v_{e t}^{2}$, and $w_{3}$ to $v_{e t}^{3}$, where $w_{1}>w_{2} \geq w_{3}$, a matrix $M$ is constructed by concatenating the vectors for each household. 
$M=\left[\begin{array}{ccc}\left(\left(w_{1} \times v_{e_{1} t_{1}}^{1}\right)+\left(w_{2} \times v_{e_{1} t_{1}}^{2}\right)+\left(w_{3} \times v_{e_{1} t_{1}}^{3}\right)\right) & \cdots & \left(\left(w_{1} \times v_{e_{1} t_{n}}^{1}\right)+\left(w_{2} \times v_{e_{1} t_{n}}^{2}\right)+\left(w_{3} \times v_{e_{1} t_{n}}^{3}\right)\right) \\ \vdots & \ddots & \vdots \\ \left(\left(w_{1} \times v_{e_{m} t_{1}}^{1}\right)+\left(w_{2} \times v_{e_{m} t_{1}}^{2}\right)+\left(w_{3} \times v_{e_{m} t_{1}}^{3}\right)\right) & \cdots & \left(\left(w_{1} \times v_{e_{m} t_{n}}^{1}\right)+\left(w_{2} \times v_{e_{m} t_{n}}^{2}\right)+\left(w_{3} \times v_{e_{m} t_{n}}^{3}\right)\right)\end{array}\right]$

In equation 3, for the matrix $\mathrm{M}$, each row represents the weighted probability of occurring an event $e$, where $e \in$ $E=\{$ shower, bathtub, clothes washer, dishwasher, irrigation $\}$ and each column represents a time $t$, where $t \in T=\{6: 00 \mathrm{am}, 6: 30 \mathrm{am}, 7: 00 \mathrm{am}, \ldots, 11: 30 \mathrm{pm}\}$, or

$t \in T=\{6: 00 \mathrm{am}, 6: 15 \mathrm{am}, 6: 30 \mathrm{am}, \ldots, 11: 30 \mathrm{pm}, 11: 45 \mathrm{pm}\}$, or

$t \in T=\{6: 00 \mathrm{am}, 7: 00 \mathrm{am}, \ldots, 11: 00 \mathrm{pm}\}$, depending on the profiling interval. Figure 3 describes the steps of TUWPU algorithm.
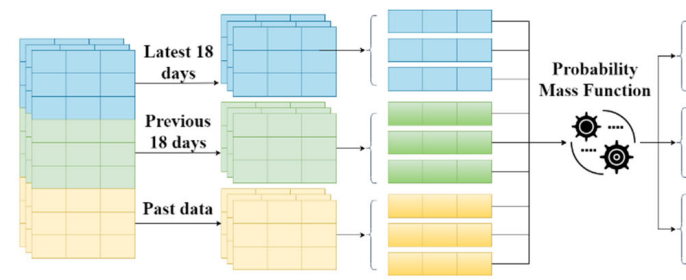

Number of

households $=$

Subsets based on
different period of different perio ime

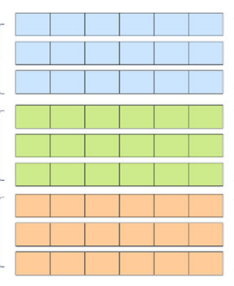

n-dimensional vector for each event for each time period

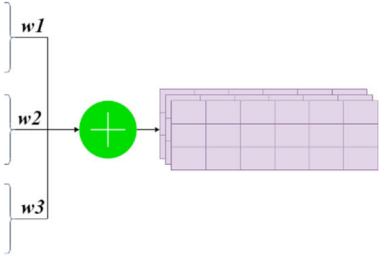

Weighted Concatenated vectors and Matrix Matrix

Figure 3. Illustration of the time of use and weighted probability of the use algorithm [20].

\subsubsection{Engineered features data set}

A feature is a numeric representation of raw data that is used as a predictor variable, which predicts an outcome variable [30]. Features are important in machine learning and data analytics, as they capture salient representations of the data and can be useful for generating new valuable insights. Feature engineering is the process of formulating suitable features using domain knowledge and the given data, model and task [30]. The success or failure of an analytics project depends highly on identifying appropriate features [31]. Therefore, feature engineering can be considered as a primary task in such projects, and a large amount of time is spent on it. Figure 4 illustrates the position of feature engineering in a typical analytics project, based on Zheng and Casari [30].

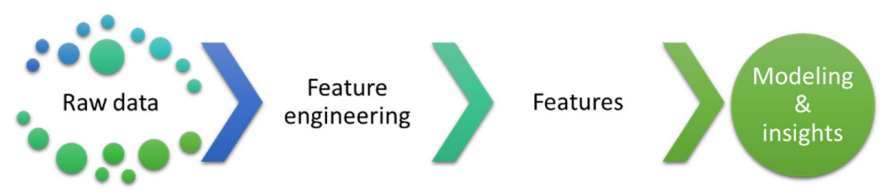

Figure 4 . The position of feature engineering in the analytics workflow.

The data collected in this study were raw in nature. Thus, to generate actionable insights, it was necessary to conduct feature engineering based on domain knowledge. First, we analysed existing literature on residential water conservation and then identified which features would be required to promote water conservation and could be used to group users based on their water-consumption data. Based on the literature, we identified several events and their attributes that were used to create new features [32-35]. Figure 5 summarises these events and their attributes. 


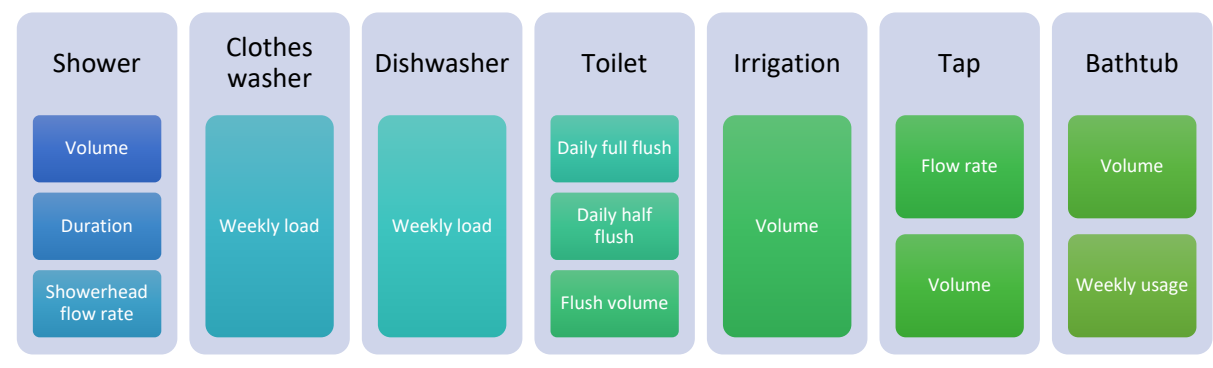

Figure 5. The events and their attributes used for feature engineering in this study.

The findings from the literature show that statistical techniques are useful in creating these features and achieving the target of water conservation by identifying users with similar consumptions. Table 4 describes the created features, creation techniques and their importance in water conservation. We discarded total water consumption/day as several studies had used this feature. Otherwise, clusters would be created based on average water consumption per day, and it would not create new patterns.

Table 4. Description of the features derived from feature engineering

\begin{tabular}{|c|c|c|c|}
\hline Event & $\begin{array}{l}\text { Derived feature } \\
\text { name }\end{array}$ & Description & Goal \\
\hline \multirow[t]{3}{*}{ Shower } & $\begin{array}{l}\text { Mean of } \\
\text { shower volume }\end{array}$ & $\begin{array}{l}\text { Mean of the volume of water used in the shower for each household } \\
\text { (litre/shower): } \\
\qquad \overline{s v}=\frac{1}{n}\left(\sum_{i=1}^{n} s v_{i}\right) \\
\text { here, } \\
\overline{s v} \text { is the mean of water used in shower, } \\
s v_{i} \text { is the volume of water used in shower } i \\
n \text { is the total number of shower events. }\end{array}$ & $\begin{array}{l}\text { Promoting water } \\
\text { conservation by } \\
\text { reducing the volume } \\
\text { of water used in the } \\
\text { shower }\end{array}$ \\
\hline & $\begin{array}{l}\text { Mean of } \\
\text { shower } \\
\text { duration }\end{array}$ & $\begin{array}{l}\text { Mean of the duration of the shower for each household (minute/shower): } \\
\qquad \overline{s d}=\frac{1}{n}\left(\sum_{i=1}^{n} s d_{i}\right) \\
\text { here, } \\
\overline{s d} \text { is the mean of shower duration, } \\
s d_{i} \text { is the duration of shower } i \\
n \text { is the total number of shower events. }\end{array}$ & $\begin{array}{l}\text { Promoting shorter } \\
\text { shower to conserve } \\
\text { more water }\end{array}$ \\
\hline & $\begin{array}{l}\text { Mean of max } \\
\text { flow for shower }\end{array}$ & $\begin{array}{l}\text { Mean of the max flow of shower for each household (litre/minute/shower): } \\
\qquad \overline{m f s}=\frac{1}{n}\left(\sum_{i=1}^{n} m f s_{i}\right) \\
\text { here, } \\
\overline{m f s} \text { is the mean of maximum water flow in shower, } \\
m f s_{i} \text { is the maximum water flow during shower } i \text {, } \\
n \text { is the total number of shower events. }\end{array}$ & $\begin{array}{l}\text { Identifying the } \\
\text { efficiency of } \\
\text { showerhead to } \\
\text { reduce water } \\
\text { consumption }\end{array}$ \\
\hline $\begin{array}{l}\text { Clothes } \\
\text { washer }\end{array}$ & $\begin{array}{l}\text { Mean of } \\
\text { weekly clothes } \\
\text { washer load }\end{array}$ & $\begin{array}{l}\text { Mean of number of weekly clothes washer loads per household (number of } \\
\text { load/week): } \\
\qquad \overline{w c l}=\frac{1}{n}\left(\sum_{i=1}^{n} w c l_{i}\right) \\
\text { here, } \\
w c l \text { is the mean of weekly clothes washer loads, } \\
w c l_{i} \text { is the of number of clothes washer loads in week } i \\
n \text { is the total number of weeks. }\end{array}$ & $\begin{array}{l}\text { Identifying the scope } \\
\text { of water conservation } \\
\text { by reducing weekly } \\
\text { clothes washer load. }\end{array}$ \\
\hline Dishwasher & $\begin{array}{l}\text { Mean of } \\
\text { weekly } \\
\text { dishwashing } \\
\text { load }\end{array}$ & $\begin{array}{l}\text { Mean of number of weekly dishwashing loads per household (number of } \\
\text { load/week): } \\
\qquad \overline{w d l}=\frac{1}{n}\left(\sum_{i=1}^{n}\left(w d l_{j}\right)\right) \\
\text { here, } \\
\overline{w d l} \text { is the mean of weekly dishwasher loads, } \\
w d l_{i} \text { is the of number of dishwasher loads in week } i \\
n \text { is the total number of weeks. }\end{array}$ & $\begin{array}{l}\text { Identifying the scope } \\
\text { of water conservation } \\
\text { by reducing weekly } \\
\text { dishwasher load. }\end{array}$ \\
\hline Toilet & $\begin{array}{l}\text { Mean of daily } \\
\text { full flush }\end{array}$ & $\begin{array}{l}\text { Mean of number of daily full flushes per household (number of full } \\
\text { flushes/day): } \\
\qquad \overline{d f f}=\frac{1}{n}\left(\sum_{i=1}^{n} d f f_{i}\right)\end{array}$ & $\begin{array}{l}\text { For discouraging the } \\
\text { usage of full flushes } \\
\text { after comparison } \\
\text { with half flushes. }\end{array}$ \\
\hline
\end{tabular}




\begin{tabular}{|c|c|c|c|}
\hline & & $\begin{array}{l}\text { here, } \\
d f f \text { is the mean of daily full flush, } \\
d f f_{i} \text { is the of number of full flushes in day } i \\
n \text { is the total number of days. }\end{array}$ & $\begin{array}{l}\text { (4:1=full flushes: } \\
\text { half flushes) }\end{array}$ \\
\hline & $\begin{array}{l}\text { Mean of daily } \\
\text { half flush }\end{array}$ & $\begin{array}{l}\text { Mean of number of daily half flushes per household (number of half } \\
\text { flushes/day/household): } \\
\qquad \overline{d h f}=\frac{1}{n}\left(\sum_{i=1}^{n} d h f_{i}\right) \\
\text { here, } \\
d h f \text { is the mean of daily half flush, } \\
d h f_{i} \text { is the of number of half flushes in day } i \\
n \text { is the total number of days. }\end{array}$ & $\begin{array}{l}\text { For encouraging the } \\
\text { usage of half flushes } \\
\text { after comparison } \\
\text { with full flushes. } \\
\text { ( } 4: 1=\text { full flushes: } \\
\text { half flushes) }\end{array}$ \\
\hline & $\begin{array}{l}\text { Mean of flush } \\
\text { volume }\end{array}$ & $\begin{array}{l}\text { Mean of the volume of water used in flush per household (litre/flush): } \\
\qquad \overline{f v}=\frac{1}{n}\left(\sum_{i=1}^{n}\left(f v_{j}\right)\right) \\
\text { here, } \\
\overline{f v} \text { is the mean of water volume used in flush, } \\
f v_{i} \text { is the of amount of water volume used in flush } i \\
n \text { is the total number flushes. }\end{array}$ & $\begin{array}{l}\text { To determine the } \\
\text { water efficiency of } \\
\text { the toilet (i.e., star) } \\
\text { To determine users } \\
\text { with a single flush } \\
\text { system }\end{array}$ \\
\hline Irrigation & $\begin{array}{l}\text { Mean of } \\
\text { irrigation } \\
\text { volume }\end{array}$ & $\begin{array}{l}\text { Mean of the volume of water used in irrigation for each household } \\
\text { (litre/irrigation): } \\
\qquad \overline{\operatorname{rrv}}=\frac{1}{n}\left(\sum_{i=1}^{n} i r r v_{i}\right) \\
\text { here, } \\
\overline{l r r v} \text { is the mean of water volume used in irrigation, } \\
i r r v_{i} \text { is the of amount of water volume used in irrigation } i, \\
n \text { is the total number irrigation events. }\end{array}$ & $\begin{array}{l}\text { To understand water } \\
\text { consumption patterns } \\
\text { in irrigation. }\end{array}$ \\
\hline \multirow[t]{2}{*}{ Tap } & $\begin{array}{l}\text { Mean of flow } \\
\text { rate }\end{array}$ & $\begin{array}{l}\text { Mean of the flow rate of tap for each household (litre/minute/tap): } \\
\qquad \overline{m f t}=\frac{1}{n}\left(\sum_{i=1}^{n} f t_{i}\right) \\
\text { here, } \\
\overline{m f t} \text { is the mean of water flow in taps, } \\
f t_{i} \text { is the water flow during tap event } i \\
n \text { is the total number of tap events. }\end{array}$ & $\begin{array}{l}\text { To determine the } \\
\text { efficiency of the tap. }\end{array}$ \\
\hline & $\begin{array}{l}\text { Mean of } \\
\text { volume }\end{array}$ & $\begin{array}{l}\text { Mean of the volume of water used in the tap for each household (litre/day): } \\
\qquad \overline{t v}=\frac{1}{n}\left(\sum_{i=1}^{n} t v_{i}\right) \\
\text { here, } \\
\overline{t v} \text { is the mean of water used in taps, } \\
t v_{i} \text { is the water used during tap event } i \text {, } \\
n \text { is the total number of tap events. }\end{array}$ & $\begin{array}{l}\text { Can be used to } \\
\text { understand tap water } \\
\text { consumption patterns }\end{array}$ \\
\hline \multirow[t]{2}{*}{ Bathtub } & $\begin{array}{l}\text { Mean of } \\
\text { Volume }\end{array}$ & $\begin{array}{l}\text { Mean of the volume of water used in bathtub for each household } \\
\text { (litre/bathtub/household): } \\
\qquad \overline{b t v}=\frac{1}{n}\left(\sum_{i=1}^{n} b t v_{i}\right) \\
\text { here, } \\
\overline{b t v} \text { is the mean of water volume used in bathtub, } \\
b t v \text { is the of amount of water volume used in bathtub event } i \text {, } \\
n \text { is the total number bathtub events. }\end{array}$ & $\begin{array}{l}\text { Can be used to } \\
\text { understand bathtub } \\
\text { water consumption } \\
\text { patterns }\end{array}$ \\
\hline & $\begin{array}{l}\text { Mean of } \\
\text { Weekly count }\end{array}$ & $\begin{array}{l}\text { Mean of the number of weekly bathtub usage per household (bathtub } \\
\text { events/week/household): } \\
\qquad \overline{w b t}=\frac{1}{n}\left(\sum_{i=1}^{n} w b t_{j}\right) \\
\text { here, } \\
w b t \text { is the mean of weekly bathtub events, } \\
w b t_{i} \text { is the of number of bathtub events in week } i \\
n \text { is the total number of weeks. }\end{array}$ & $\begin{array}{l}\text { Can be used to } \\
\text { understand bathtub } \\
\text { usage frequency }\end{array}$ \\
\hline
\end{tabular}

\subsection{Experiments}

Because the types and goals of the two data sets are different, we applied two different approaches. As the EF data set is less complex and easier to explain, summarise and visualise, therefore, we first determined the optimal number of clusters for this data set using the elbow method [24] and k-means clustering [36, 37]. Before determining the optimal number of clusters, a dimension reduction technique was applied on the data set. Later, we applied k-means [36, 37], affinity propagation [38], mean shift [39], spectral [40], birch [41] and hierarchical 
agglomerative clustering [42] techniques to compare their performance in terms of running time and clustering quality. On the other hand, the TUWPU data set is more complex than the EF data set as it provides outputs at three different intervals (i.e., 15 minutes, 30 minutes and 60 minutes) for five different water end-use categories (shower, bathtub, washing machine, dishwasher, gardening) for different day types (i.e., weekday, weekend). As representing data items in a hierarchy is beneficial to data summarisation and visualisation, therefore, we chose hierarchical agglomerative clustering [42] for the TUWPU data set, as this method forms clusters iteratively by dividing the patterns using a bottom-up approach [8]. In addition, we applied different linkage methods and compared their performance to identify the suitable one for hierarchical agglomerative clustering. The following subsection discusses these approaches and their applications in our scenario briefly.

\subsubsection{Clustering the EF data set}

Determining the appropriate number of clusters is an important task as it controls the granularity of cluster analysis and some clustering algorithms require that parameter [24]. One of the popular and simple methods is to determine the number of clusters as $\sqrt{\frac{n}{2}}$ for a data set with $n$ points. However, this method would provide a greater number of clusters for a big data set. Therefore, we chose another popular method called the elbow method to determine the appropriate number of clusters. First, we applied a dimension reduction technique and then applied the elbow method with the k-means clustering technique and identified the optimal number of clusters. Subsequently, we applied other clustering techniques to compare the clustering quality and run time. As the ground truth was not available, instead of using extrinsic methods, we applied two intrinsic methods, namely silhouette coefficient and the Calinski-Harabasz index. After determining the best clustering technique, we identified the traits of each cluster.

Dimension reduction is the process of reducing the number of attributes while preserving as much possible variance in the original data set as possible. In this experiment, we applied principal component analysis (PCA) as the dimension reduction technique. Principal Component Analysis (PCA) is an algorithm that minimises the dimension of the data set by preserving most of the variance [43]. This task is accomplished by identifying the dimensions that explain the maximum variance in the data [44]. In PCA, new variables known as principal components are constructed and are linear combinations of the initial variables. The first principal component explains the largest proportion of the variance; the second principal component explains the second largest proportion, and so on. Therefore, a smaller number of principal components leads to lower variance. This is why, to choose the optimal number of principal components, $95 \%$ explained variance should be used instead of total variance [43]. After applying the dimension reduction technique, we chose the k-means clustering algorithm with the elbow method to determine the optimal number of clusters.

$k$-means clustering is one of the most well-known algorithms and is easy to understand and easy to implement [36,37]. The algorithm divides the data set into $k$ groups, where the value of $k$ is defined by a user. Each $k$ presents the centroid of a cluster and is typically assigned randomly [9]. The objective function $J$ is defined in Equation 4.

$$
\text { minimise } J=\sum_{j=1}^{k} \sum_{i=1}^{n}\left\|x_{i}^{(j)}-c_{j}\right\|^{2}
$$

where $\left\|x_{i}^{(j)}-c_{j}\right\|^{2}$ is the distance between a data point $x_{i}^{(j)}$ and centroid $c_{j}$ [8]. The instances within the same group are similar to each other, and instances from different groups are different from each other [24]. Therefore, we chose this algorithm to identify households with similar water-consumption patterns. However, determining the optimal numbers for $k$ is a difficult task for this algorithm. To overcome this challenge, we used the elbow method [24].

The elbow method is based on the finding that the sum of within-cluster variance for each cluster decreases with an increasing number of clusters [24]. This occurs because having more clusters helps to identify more groups of items with similar characteristics. However, with too many clusters, there might not be enough of a reduction in the sum of within-cluster variances because partitioning a well-integrated cluster into two clusters provides a reduction by a very small margin. Therefore, by plotting the curve of the sum of within-cluster variances for different values of $k$, where $k>0$, the correct number of clusters can be identified from the first or most significant turning point of the curve. This process is defined mathematically in Equation 5.

$$
\operatorname{minimise}\left(\sum_{k=1}^{K} W\left(C_{k}\right)\right)
$$


where $C_{k}$ is the $k^{t h}$ cluster, and $W\left(C_{k}\right)$ is the within-cluster variation. The sum of within-cluster variation $W\left(C_{k}\right)$ is defined in Equation 6.

$$
W\left(C_{k}\right)=\sum_{x_{i} \in C_{k}}\left(x_{i}-\mu_{k}\right)^{2}
$$

where $x_{i}$ is a data point that belongs to the cluster $C_{k}$, and $\mu_{k}$ is the mean of points assigned to the cluster $C_{k}$. Equation 7 represents the final equation.

$$
\operatorname{minimise}\left(\sum_{k=1}^{K} \sum_{x_{i} \in C_{k}}\left(x_{i}-\mu_{k}\right)^{2}\right)
$$

We observed that for the EF data set, four principal components explained $95 \%$ of the variance in the data set. Figure 6 shows each number of principal components and its corresponding ratio of explained variance.

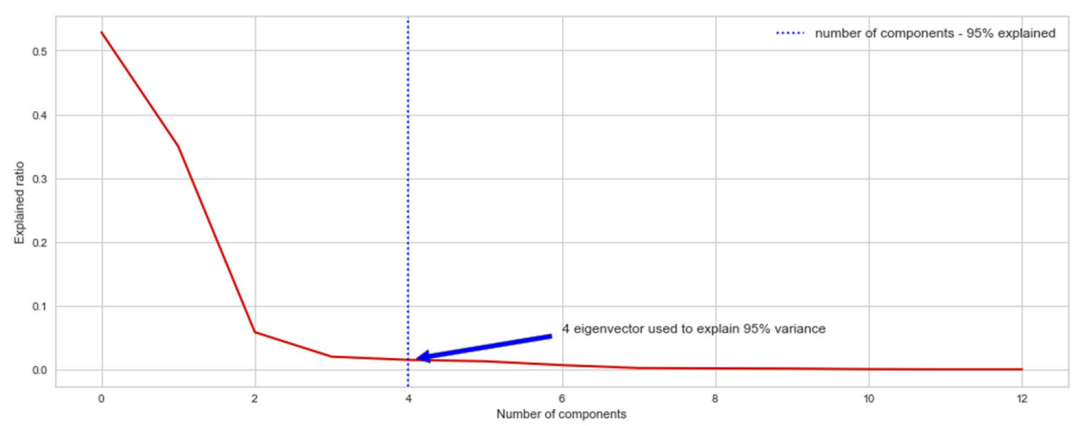

Figure 6. Ratio of explained variance for the different number of principal components for the EF data set.

After identifying the optimal number of principal components, we applied the elbow method to determine the optimal number of clusters. The elbow method used in this study was implemented by selecting the optimal number of clusters by using k-means clustering with a varying number of clusters. After calculating the sum of within-cluster variances for different values of $k$ with four principal components and clustering model k-means, where $0<k<20$, we found that $k=5$ is the optimal number of clusters, as the most significant turning point occurred there. Figure 7 plots the sum of the within-cluster variances for different values of $k$ after PCA was performed. From the figure, we concluded that the number of optimal clusters is five as the elbow appears there.

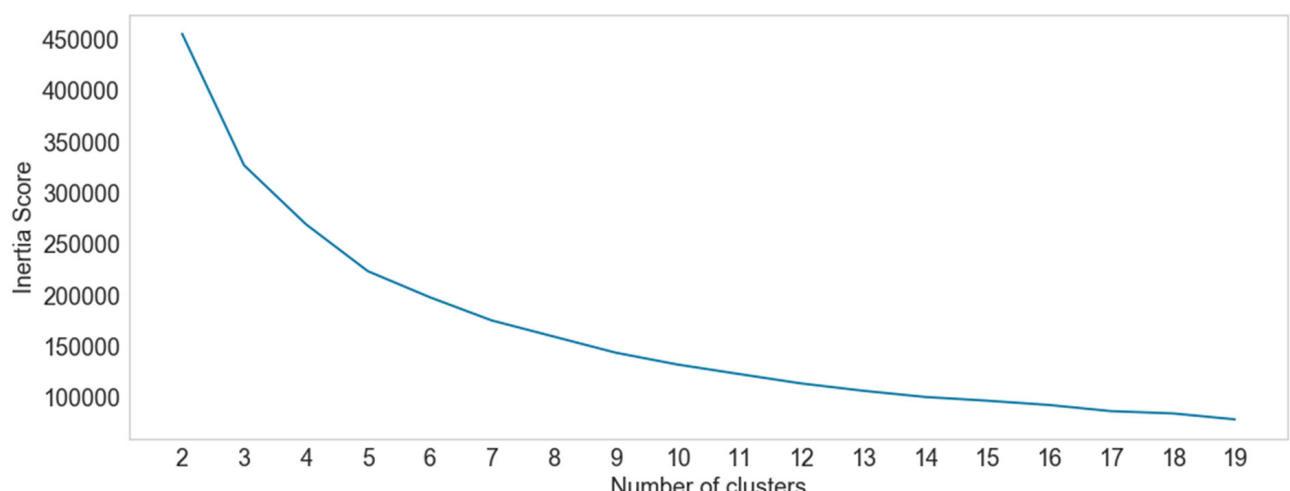

Figure 7. Plotted curve of the within-cluster variances for different values of $k$ after PCA was performed

For further confirmation regarding the optimal value of $k$, we applied a silhouette plot for different values of $k$. Silhouette plots display the silhouette coefficient for each data point from the clusters. Figure S2 displays the silhouette plots of k-means for 306 households in 3,4,5 and 6 clusters. From these plots, we concluded between cluster 4 and 5 in figure S2 (b) and S2 (c); cluster 5 would be the optimal number of clusters as households were more uniform among the clusters compared to cluster 4. 
After identifying the optimal number of clusters, for the next step, we applied different clustering techniques on the transformed EF data set. Besides k-means, other clustering techniques included affinity propagation [38], mean shift [39], spectral [40], birch [41] and hierarchical agglomerative clustering [42]. In affinity propagation, clusters are created by sending messages between data points until convergence [42]. Mean shift is a centroid-based algorithm that aims to find blobs in a smooth density of data points [39, 45]. Spectral clustering performs dimension reduction before clustering in fewer dimensions by using the eigenvalues of the similarity matrix of data points [45]. On the other hand, the birch clustering technique works by building a clustering feature tree (CFT) for the data points. Lastly, in hierarchical agglomerative clustering, initially, all the data items are considered separate clusters, and they iteratively merge with one of the closest clusters until there is only one larger cluster, which becomes the hierarchy's root. After running these algorithms on the transformed EF data set, we recorded the run time to measure the performance in terms of running time. In addition, we also checked the scalability of these algorithms by executing them in different sizes to get an average-case performance. Furthermore, as the ground truth labels for the transformed EF data set were not available, therefore, to measure the clustering quality, the silhouette coefficient and Calinski-Harabasz indexes were recorded for each technique. The silhouette coefficient is defined for each sample using two scores $\mathrm{a}, \mathrm{b}$ and silhouette coefficient $\mathrm{s}$ can be defined using equation 8 .

$$
s=\frac{b-a}{\max (a, b)}
$$

Here, $a$ is the mean distance between one sample and other points from the same class, and $b$ is the mean distance between one sample and other points from the next nearest cluster.

Calinski-Harabasz index, also known as the Variance Ratio Criterion, is the ratio of the sum of betweencluster dispersion and of within-cluster dispersion for all clusters [45]. It can be defined using Equation 9.

$$
s=\frac{\operatorname{tr}\left(B_{k}\right)}{\operatorname{tr}\left(W_{k}\right)} \times \frac{n_{e}-k}{k-1}
$$

where $\mathrm{E}$ is the data set, $n_{e}$ is the size of the data set, $k$ is the number of clusters, $\operatorname{tr}\left(B_{k}\right)$ and $\operatorname{tr}\left(W_{k}\right)$ are the trace between the group dispersion matrix and within-cluster dispersion matrix, respectively.

After completing the experiment, we observed that performance and clustering quality metrics varied depending on the clustering technique. For instance, in terms of performance in time taken to cluster, agglomerative clustering took the least amount of time, and the mean shift took the greatest amount of time. For measuring the quality of clustering, in the case of silhouette score, the mean shift outperformed other techniques followed by affinity propagation and k-means, and when using the Calinski-Harabaz index, k-means provided the best result. Table 5 presents the data collected from this experiment. After looking carefully at the data, we noticed that even though the mean shift technique performed better with the silhouette score, however, it performed poorly in terms of time taken and when using the Calinski-Harabaz index. K-means provided the best result with the Calinski-Harabaz index score, third-best with the silhouette score and average in terms of time performance. It seemed that k-means would be a comparatively better choice in terms of these matrices. However, before taking the final decision, we also observed the scalability of these techniques.

Table 5. Performance and clustering quality metrics of different clustering techniques for the EF data set

\begin{tabular}{|l|c|c|c|}
\hline Clustering Algorithm & $\begin{array}{l}\text { Time taken } \\
\text { (in seconds) }\end{array}$ & Silhouette score & Calinski-Harabaz index score \\
\hline K-means & 0.048 & 0.2999 & $\mathbf{1 5 6 . 6 2 4 2}$ \\
\hline Affinity Propagation & 0.140 & 0.3061 & 153.5803 \\
\hline Mean Shift & 0.970 & $\mathbf{0 . 4 4 7 8}$ & 81.0378 \\
\hline Spectral Clustering & 0.046 & 0.2514 & 130.5797 \\
\hline Birch & 0.020 & 0.2776 & 132.8396 \\
\hline Agglomerative Clustering & $\mathbf{0 . 0 0 4 s}$ & 0.2549 & 129.9911 \\
\hline
\end{tabular}

To understand the scaling performance of the clustering techniques for the EF data set, we ran experiments several times for different data sizes. Initially, we started with 10 households, and with a step size of 10, we completed all the 306 households. Figure 8 represents the result of the experiment by drawing a point at the mean of the time taken to create clusters and the error bar. From there, we concluded that mean shift was the worst-performing clustering technique in terms of scaling performance, and k-means showed good performance. 
As detailed earlier, we identified that k-means performed better in clustering quality and it was also scalable; therefore, we decided to further refine the clusters using k-means clustering.

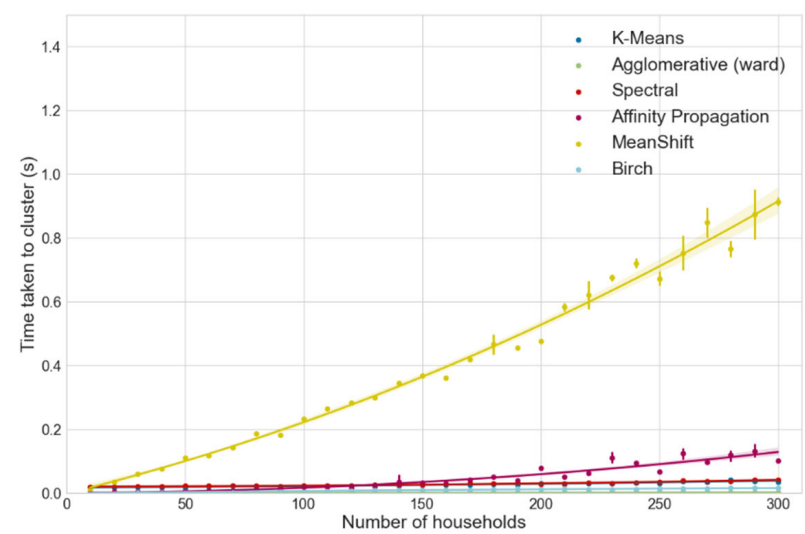

Figure 8. Scaling performance of different clustering techniques for the EF data set.

After performing clustering on the EF data set using the k-means algorithm, we moved to the TUWPU data set to group users based on their consumption time for each event. To accurately interpret the result, we deployed hierarchical agglomerative clustering (HAC) because representing data items as a hierarchy is beneficial to data summarisation and visualisation [24]. The following subsection discusses the method in detail.

\subsubsection{Clustering the TUWPU data set}

Hierarchical agglomerative clustering is a variation on hierarchical clustering where the hierarchical decomposition is performed in either a bottom-up or a top-down approach [24]. In this method, initially, all the data items are considered separate clusters, and they iteratively merge with one of the closest clusters until there is only one larger cluster, which becomes the hierarchy's root. After measuring the distance between two clusters, a linkage criterion is used to merge the two clusters into one. In this study, to measure the distance between two clusters, we used Euclidean distance as Equation 8.

$$
d(p, q)=\sqrt{\sum_{i=1}^{n}\left(p_{i}-q_{i}\right)^{2}}
$$

where $p$ and $q$ are two points in Euclidean $n$-space.

Linkage methods determine the distance between clusters as a function of pairwise distances between data points [46]. Several linkage methods are currently available, each with its own characteristics. We explored most of the common linkage methods and determined the most suitable method by comparing the clustering quality metrics and dendrograms generated by HAC. The details of these methods, along with their notations, are discussed in this section.

Table 5. Notations and their descriptions for different linkage methods.

\begin{tabular}{ll}
\hline Notation & Description \\
\hline$d_{m j}$ & Distance between clusters $m$ and $j$ \\
$m$ & Merged cluster \\
$d_{k j}$ & Distance between clusters $k$ and $j$ \\
$d_{l j}$ & Distance between clusters $l$ and $j$ \\
$d_{k l}$ & Distance between clusters $k$ and $l$ \\
$N_{j}$ & Number of data items in cluster $j$ \\
$N_{k}$ & Number of data items in cluster $k$ \\
$N_{l}$ & Number of data items in cluster $l$
\end{tabular}


Single-linkage method: In this method, the distance between two clusters is the minimum distance between one item from one cluster and another item from another cluster [47]. After the distance between all clusters is calculated, the two closest clusters are merged into one larger cluster. However, one of the limitations of this method is that it suffers from the chaining effect and has the tendency to produce irregular or widened clusters [47, 48]. Equation 9 can be used to calculate the minimum distance.

$$
d_{m j}=\min \left(d_{k j}, d_{l j}\right)
$$

Complete-linkage method: In the complete-linkage method, the distance between two clusters is the maximum distance among all pairwise distances between observations in the two clusters [47]. This method can produce tightly bound or compact clusters [49]. Equation 10 can be used to determine the maximum distance.

$$
d_{m j}=\max \left(d_{k j}, d_{l j}\right)
$$

Average-linkage method: In this method, the distance between two clusters is the average distance between the pairs of observations in the two clusters [50]. Equation 11 can be used to derive this average distance.

$$
d_{m j}=\frac{N_{k} d_{k j}+N_{l} d_{l j}}{N_{m}}
$$

Ward's linkage method: The aim of Ward's linkage method is to minimise the total within-cluster variance. To this end, a pair of clusters to be merged on each iteration is chosen such that it produces the minimum within-cluster variance. Here, the distance between two clusters is the sum of squared deviations from the observations to the centroids [50] and can be calculated using equation 12 .

$$
d_{m j}=\frac{\left(N_{j}+N_{k}\right) d_{k j}+\left(N_{j}+N_{l}\right) d_{l j}-N_{j} d_{k l}}{N_{j}+N_{m}}
$$

Clustering may vary depending on the profiling interval and linkage method used. Figure 9 shows the dendrograms of the shower event during weekdays with the 30-minute interval for different linkage methods. From that figure, we can see that the number of clusters varies depending on the linkage methods as the distance cut-off value differs. Therefore, after applying the above linkage methods on the TUWPU data set, we measured the clustering quality using the silhouette coefficient and the Calinski-Harabasz index as well as the distance cut-off value to determine the best profiling interval and linkage method.

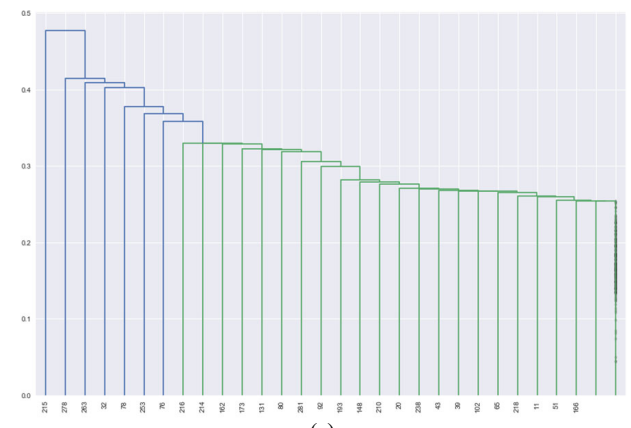

(a)

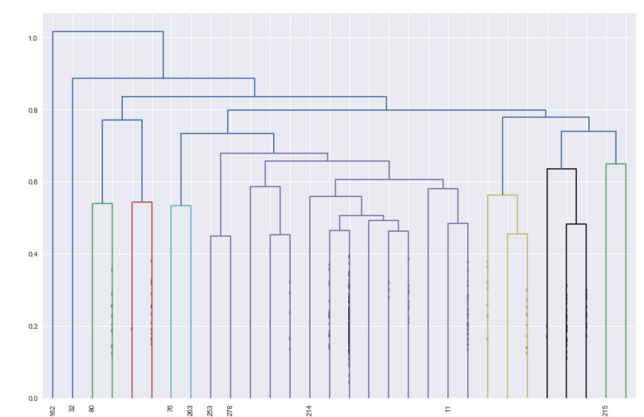

(b) 


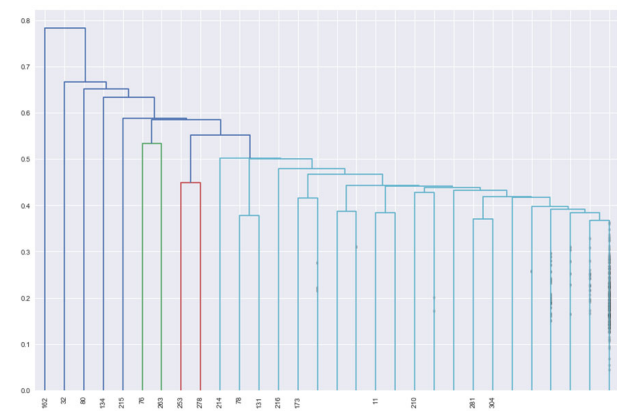

(c)

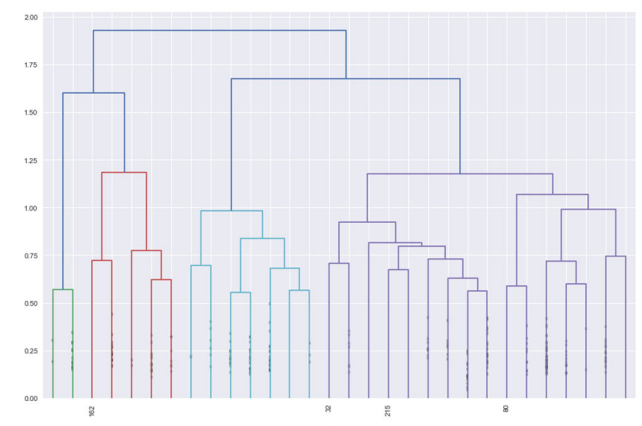

(d)

Figure 9. Dendrograms of the shower event during weekdays with the 30-minute interval for a) single linkage, b) complete linkage, c) average linkage, d) Ward's linkage. Here, the x-axis represents households, and the y-axis represents the distance.

After performing the experiments, all results were collected and analysed carefully. The following section discusses the results and findings.

\section{Results and Discussion}

The previous sections described the comprehensive experiments on the two data sets derived from residential DWMs. This section presents our findings and discusses their implications for promoting water-conscious behaviour. To visualise the outcomes of this study, we have applied various visualisation tools such as Power BI [51] and Seaborn [52].

\subsection{Engineered features data set: Findings and discussion}

Identifying the optimal number of clusters and traits for each cluster is beneficial for water utility companies to better understand their customers and devise effective water-conservation awareness programs. To this end, after applying the $k$-means clustering algorithm to the $\mathrm{EF}$ data set, we identified the optimal number of clusters to be five and determined the characteristics of each cluster. Among the 306 households, the number of households in each cluster varies. For instance, Clusters 1, 2, 3, 4 and 5 contain $61,124,59,15$ and 47 households, respectively. Figure 10 illustrates the number of households in each cluster.

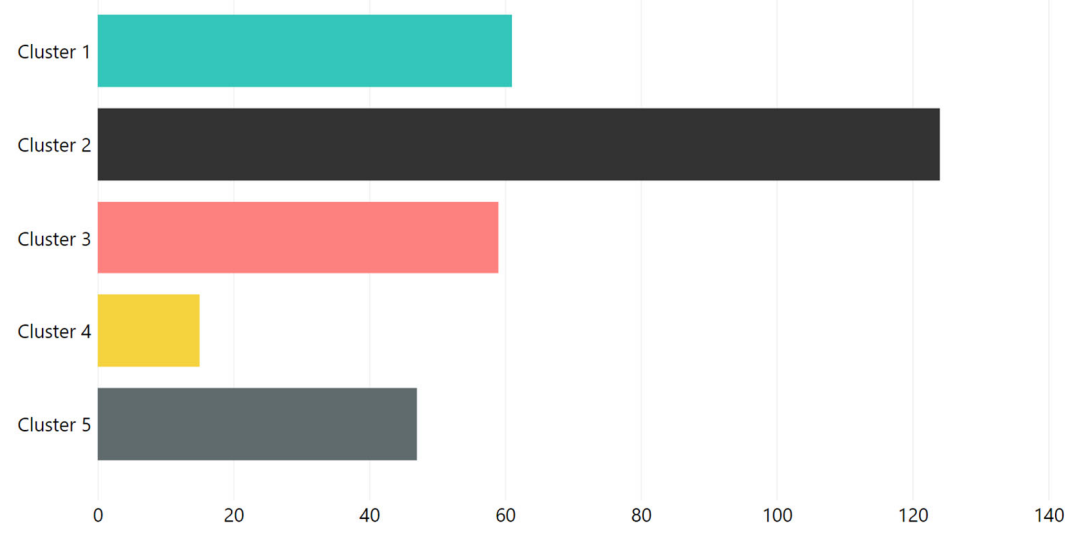

Figure 10. The number of households by cluster for the EF data set.

After determining the optimal number of clusters from the EF data set, we investigated the characteristics of each cluster and found some significant, valuable and previously unseen insights. To summarise the results, we applied summary statistics on each feature. Summary statistics are commonly used to present the largest amount 
of information in the simplest possible manner. In this study, we employed a five-number summary that provides values for the median, first quartile, third quartile, minimum and maximum based on the observations of a sample. These values can be used to quickly compare several sets of observations. In addition, we also calculated the arithmetic mean of these features to understand and report central tendencies. Table 6 presents the arithmetic mean of different events for each cluster to denote their characteristics.

Table 6. Summary of the traits of clusters based on the arithmetic mean of different events

\begin{tabular}{|c|c|c|c|c|c|c|}
\hline \multirow{2}{*}{ Event } & \multicolumn{5}{|c|}{ Clusters } & \multirow{2}{*}{ Feature } \\
\hline & Cluster 1 & Cluster 2 & Cluster 3 & Cluster 4 & Cluster 5 & \\
\hline \multirow{3}{*}{ Shower } & 40.19 & 47.37 & 47.79 & 44.67 & 55.63 & $\begin{array}{l}\text { Mean of shower } \\
\text { volume }\end{array}$ \\
\hline & 5.68 & 5.66 & 5.89 & 5.51 & 5.83 & $\begin{array}{l}\text { Mean of shower } \\
\text { duration }\end{array}$ \\
\hline & 8.83 & 10.82 & 10.54 & 10.70 & 11.93 & $\begin{array}{l}\text { Mean of max flow for } \\
\text { shower }\end{array}$ \\
\hline \multirow{3}{*}{ Toilet } & 8.48 & 8.14 & 14.29 & 20.73 & 8.15 & Mean of daily full flush \\
\hline & 6.44 & 6.06 & 12.20 & 21.93 & 6.57 & $\begin{array}{l}\text { Mean of daily half } \\
\text { flush }\end{array}$ \\
\hline & 5.33 & 5.36 & 5.24 & 5.40 & 5.21 & Mean of flush volume \\
\hline $\begin{array}{l}\text { Clothes } \\
\text { washer }\end{array}$ & 2.95 & 2.95 & 3.46 & 6.40 & 2.68 & $\begin{array}{l}\text { Mean of weekly clothes } \\
\text { washer load }\end{array}$ \\
\hline Dishwasher & 3 & 3 & 6 & 8 & 3 & $\begin{array}{l}\text { Mean of weekly } \\
\text { dishwashing load }\end{array}$ \\
\hline \multirow[t]{2}{*}{ Tap } & 4.13 & 4.33 & 4.53 & 4.49 & 4.55 & Mean of flow rate \\
\hline & 47.02 & 41.40 & 87.02 & 161.68 & 41.76 & Mean of volume \\
\hline Bathtub & 0.82 & 1.98 & 4.14 & 4.53 & 3.72 & Weekly count \\
\hline Irrigation & 190 & 190 & 190 & 160 & 170 & $\begin{array}{l}\text { Mean of irrigation } \\
\text { volume }\end{array}$ \\
\hline
\end{tabular}

Table 6 shows that several aspects are distinctive between these clusters. For instance, Cluster 1 can be differentiated from other clusters by its lower mean of shower volume and lower mean of maximum shower flow. In contrast, Cluster 5 has the highest value of mean shower volume and maximum shower flow. Another relevant finding is that the mean of daily flushes, the volume of tap water used, and the number of weekly clothes-washer loads for Cluster 4 is the highest compared to other clusters. Even though socio-demographic data are not available for this study, we assume based on previous studies that households with elderly people and infants might fall in this cluster.

Another finding from the results is the relationship between the number of flushes and tap-water volume. We observed that households with a higher number of daily flushes tend to use more water from taps. Figure 11 shows that Cluster 4 has the highest number of average daily full and half flushes as well as the highest amount of tap water compared to other clusters. On the other hand, Cluster 2 has the lowest number of full flushes and used the least amount of tap water.

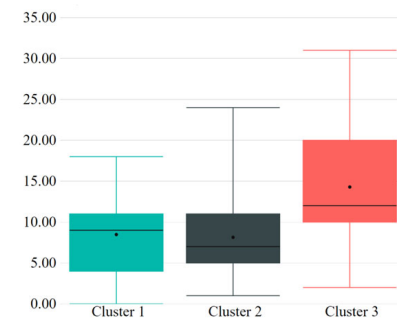

(a)

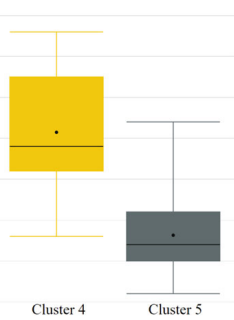

Cluster 5

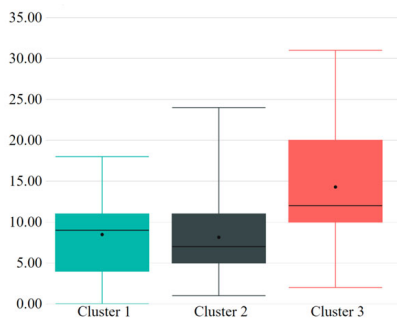

(b)

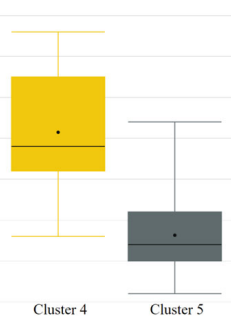

Cluster $4 \quad$ Cluster 5 


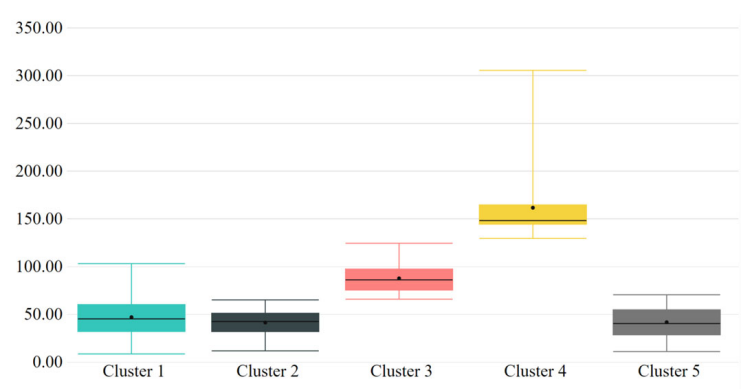

(c)

Figure 11. Comparison among different clusters in terms of (a) mean of daily full flushes, (b) mean of daily half flushes and (c) mean of tap water used daily.

This might not be surprising at first because people typically use tap water to wash their hands after using the toilet. However, during the current and unprecedented global outbreak of the novel coronavirus disease 2019 (COVID-19), insights from tap-water usage can be used to understand the adaptation of hand-hygiene practices among the population to prevent infection from the COVID-19 virus [53]. For instance, the water consumption patterns of tap-water usage from before and during the pandemic could be compared to determine the acceptance of hand hygiene among different groups of consumers with the inclusion of socio-demographic data and could be useful to policymakers and health experts in deciding their next actions. For instance, after performing clustering on the tap-water usage data collected before and during the pandemic, the difference in the mean of the tap water used daily among the clusters could be used to help understand changes in hand hygiene practices. As the data set used in this study was collected before (i.e., 2010) the COVID-19 pandemic, it is not possible to undertake changes in this present study. Through applying our approach with a 2020 high resolution data set, this type of evaluation could be conducted in the future.

\subsection{Time of use and weighted probability of use data set: Findings and discussion}

The TUWPU data set addresses the aspects of demand management by characterising the patterns of behaviour and habits of water consumption by providing the probability that a specific water-use event will occur at a particular time, for various intervals throughout the day. With the application of HAC, the results from this data set show that the participating households fall under different clusters based on the type of day, event and interval. For instance, for the shower event, one household can be in different clusters for its behaviours and habits depending on the type of the day (i.e., weekday or weekend) and profiling interval (15-minute, 30-minute or 60minute). Figure 12 shows the result of applying HAC on 306 households for the shower event and the three profiling intervals.

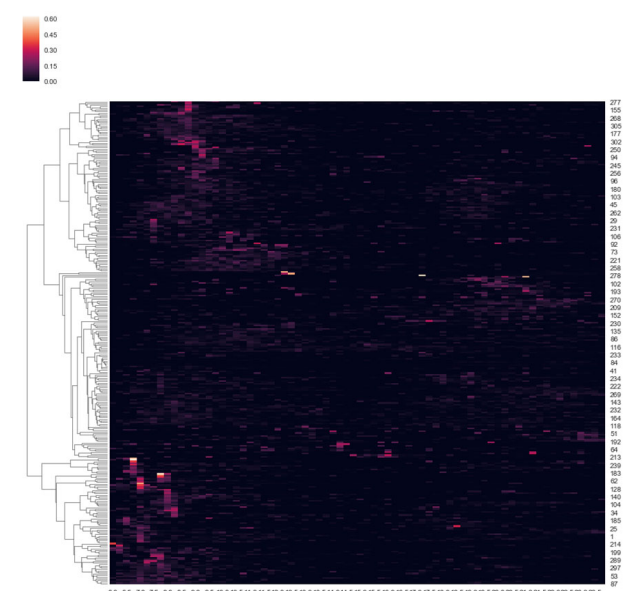

(a)

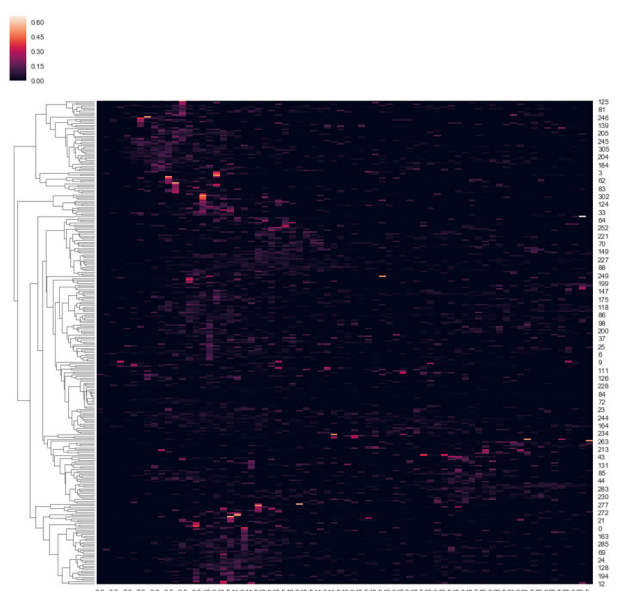

(b) 


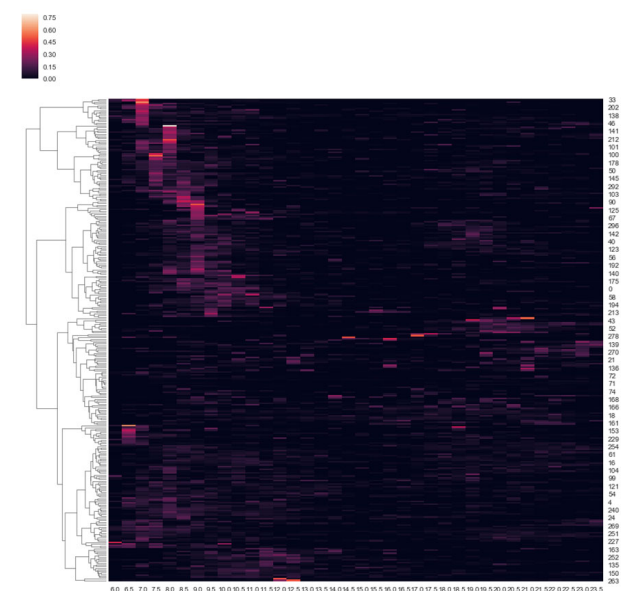

(c)

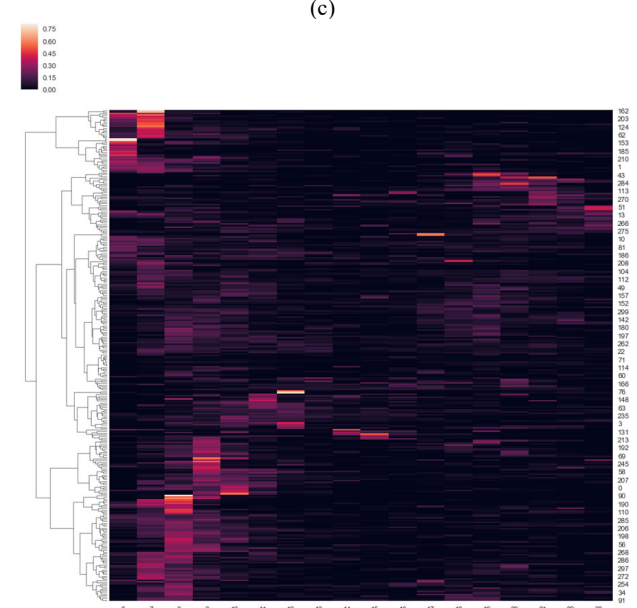

(e)

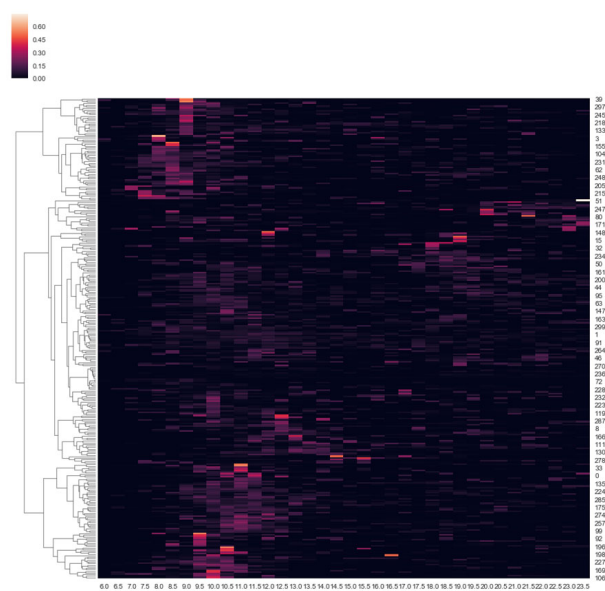

(d)
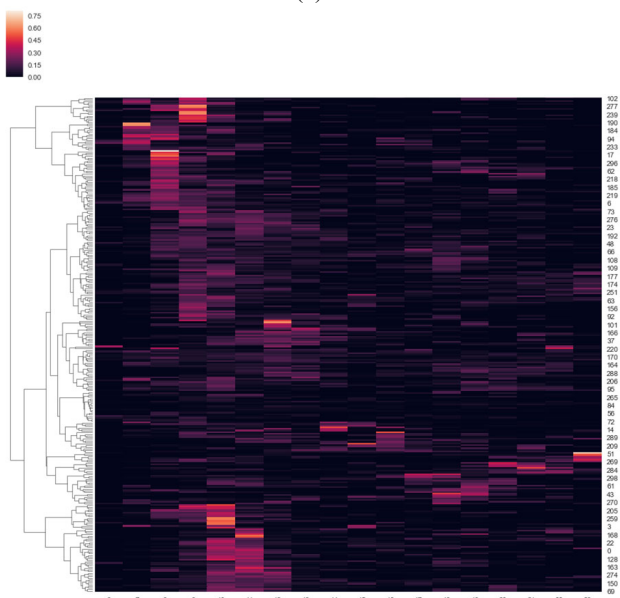

(f)

Figure 12. Result of applying HAC to 306 households for the shower event at a) a 15-minute interval on weekdays, b) a 15-minute interval on weekends, c) a 30-minute interval on weekdays, d) a 30-minute interval on weekends e) a 60-minute interval on weekdays and f) a 60-minute interval on weekends. Here, the $\mathrm{x}$-axis

represents the times for different intervals and the $y$-axis represents the household numbers.

The heat map associated with the dendrograms represents the intensity of the probability of a shower occurring in a household at a particular time. A higher probability represents a strong habit that is a higher chance of the same event reoccurring at that particular time. From these dendrograms, it is possible to identify households with similar consumption patterns that fall under the same clusters, and the intensity or magnitude of a habit occurring at a particular time. Such clustering based on the probability of an event occurring at a particular time can be valuable to identify households with similar patterns, and this information can be used for demand management. For instance, a recommendation to shift an event outside of the hours of peak demand can be made through a personalised recommender system because shifting the timing of water consumption on the customer end presents a viable cost-reduction opportunity for water utility companies [54]. However, making recommendations to shift an event time by 60 minutes might not be effective from the perspective of behaviour theory. In this case, profiling even at a 15-minute interval can identify a higher probability of an event occurring at a particular time; therefore, recommendations can be made to shift event time by 15 minutes. However, depending on the objectives of the utilities and types of events they are interested in examining, a 30-minute or 60-minute profiling interval can be chosen for clustering. For such scenarios, the dendrograms generated from HAC provide the flexibility to determine the number of clusters after performing the analysis. The number of optimal clusters can be determined based on the distance between the two newly merged clusters, where a gap in 
the distance means that the two clusters do not belong in one cluster. In other words, a larger distance means a greater difference between the two clusters for the chosen features. However, there is no golden rule that will determine the optimal number of clusters from dendrograms. The results could vary based on the nature of the problem and the data set. Therefore, for the TUWPU data set, we set the distance cut-off value at $70 \%$ from the final merge to understand the differences based on the event, type of day and profiling interval. Table 7 presents a summary of the number of clusters for different events at the three different profiling intervals.

Table 7. Estimated number of clusters for different events, type of day at different profiling interval

\begin{tabular}{|c|c|c|c|c|}
\hline \multirow{2}{*}{ Event } & \multirow{2}{*}{ Type of day } & \multicolumn{3}{|c|}{ Number of clusters for interval } \\
\cline { 3 - 5 } & & $\mathbf{1 5}$ min & $\mathbf{3 0}$ min & $\mathbf{6 0}$ min \\
\hline \multirow{2}{*}{ Shower } & Weekday & 5 & 4 & 3 \\
\cline { 2 - 5 } & Weekend & 6 & 3 & 3 \\
\hline Clothes washer & Weekday & 7 & 5 & 4 \\
\cline { 2 - 5 } & Weekend & 5 & 5 & 5 \\
\hline Dishwasher & Weekday & 6 & 2 & 3 \\
\cline { 2 - 5 } & Weekend & 3 & 4 & 3 \\
\hline \multirow{2}{*}{ Bathtub } & Weekday & 4 & 3 & 3 \\
\cline { 2 - 5 } & Weekend & 5 & 4 & 4 \\
\hline Irrigation & Weekday & 4 & 4 & 6 \\
\cline { 2 - 5 } & Weekend & 6 & 5 & 5 \\
\hline
\end{tabular}

In this experiment, we also sought to determine the most suitable profiling interval among the three intervals. The distance cut-off value can be a good measure for this task, as previously mentioned since a larger distance means a greater difference between the two clusters based on the chosen features. Based on this property, we have examined the distance cut-off values for these three profiling intervals and observed that the 15-minute profiling interval performs merging at the lowest-distance cut-off values compared to the two other intervals. This indicates that the households in each cluster for the 15-minute interval are more similar than those in the two other intervals. We also observed that when using the silhouette coefficient, the average linkage method outperformed other linkage methods at a 15-minute profiling interval. Conversely, when using the Calinski-Harabasz index, we observed that Ward's method outperforms the other linkage methods. This finding aligns with a previous study, where Ward's method also outperformed other linkage methods [55]. Table S1 represents the clustering quality analysis for different linkage methods at different intervals. As two linkage methods performed better at two intervals in two metrics, therefore, to determine the best one, we investigated the characteristics of these two metrics. We observed that the silhouette coefficient provides a score between -1 and +1 , where a score towards 1 represents incorrect clustering, and a score towards +1 represent highly dense clustering. For the CalinskiHarabasz index, a higher value represents better-defined clusters. For the TUWPU data set at a 60 -minute profiling interval, the Calinski-Harabasz index scores were not as high to represent better-defined clusters. As the silhouette coefficient provides a better understanding of the quality of clustering for the TUWPU, therefore, we concluded that a 15-minute profiling interval is the best profiling interval and the average linkage method is the best linkage method for the TUWPU data set. Therefore, we conclude that the 15-minute profiling interval outperforms the 30-minute and 60-minute profiling intervals. Figure 13 shows a sample comparison between different profiling intervals for the shower event on weekdays. From this figure, we can conclude that the number of clusters varies depending on the profiling interval, and the 15-minute profiling interval provides the best result since it merges at the lowest-distance cut-off values. From this type of dendrogram, it is possible to identify groups of households that have a similar probability of an event occurring at a particular time, which has implications for improved water demand management and profiling. 


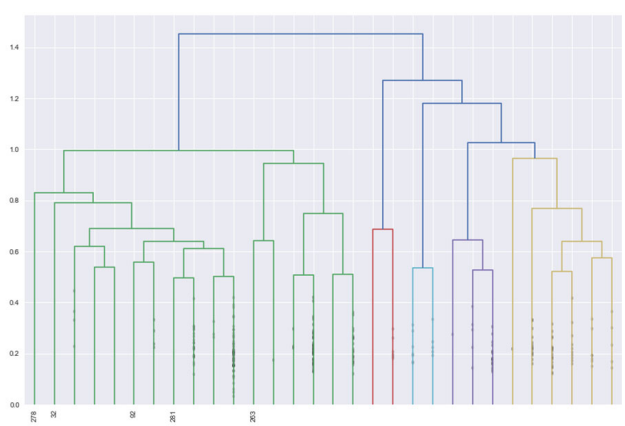

(a)

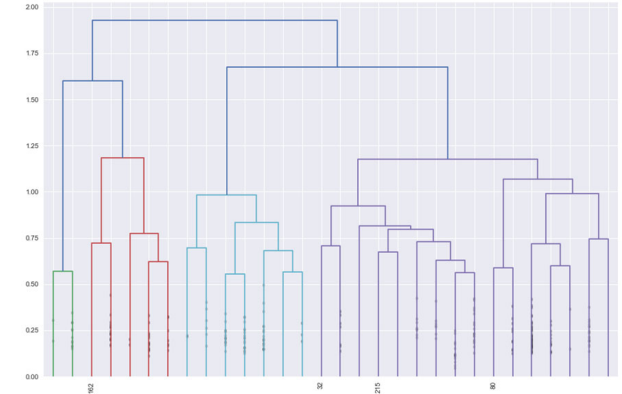

(b)

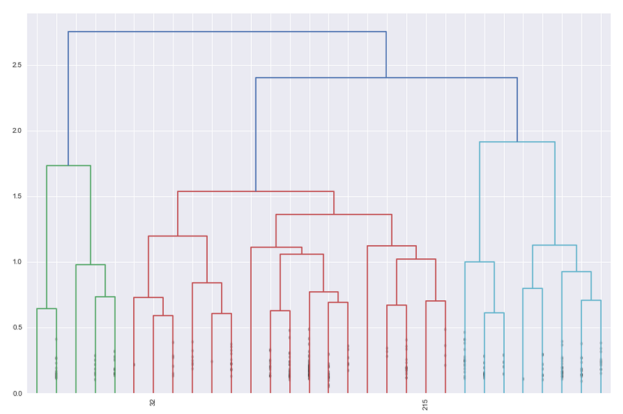

(c)

Figure 13. Comparison among different profiling intervals for the shower event on weekdays using dendrograms: a) 15minute profiling interval, b) 30-minute profiling interval and c) 60 -minute profiling interval.

The insights found from the TUWPU data set after performing HAC might be valuable to water utility companies, researchers and policymakers in better understanding the behaviours and habits for the given sample. Such insights can be used to determine more effective water-conservation programs, as well as manage demand to reduce operational costs and improve the overall efficiency of the system. Furthermore, the outcomes from this study can be used in a recommender system for promoting water-conscious behaviours, by identifying households with similar patterns and suggesting activities that are popular among their neighbours (i.e., similar households).

\section{Conclusions and Future Work}

Water scarcity is an ongoing concern for many cities, placing ever more importance on water conservation. Water utilities are facing a significant challenge to ensure that there is enough water for everyone during prolonged drought. In addition, efficiently providing sufficient water pressure during hours of peak demand is another challenge that troubles some utility companies. Data collected from DWMs allow them to overcome these challenges. However, understanding consumers and their water usage behaviour are essential in devising effective strategies for water conservation and demand management.

In this study, we have performed a comprehensive analysis of residential DWM data by clustering consumers by consumption patterns. Two data sets were prepared, and clustering techniques were applied based on the nature of the data and the objective of the clustering. For the EF data set, we found that the optimal number of clusters is five and k-means is the most suitable clustering technique, thus we applied the k-means clustering technique. Later, we discussed the traits of these clusters. For the TUWPU data set, we applied HAC using different linkage methods and found that the optimum number of clusters varies based on the profiling interval, type of waterconsumption event and type of day (i.e., weekday or weekend). We also observed that 15-minute profiling is the most suitable profiling interval in terms of forming clusters based on the similarity of consumer behaviour. The study findings would be beneficial to policymakers and researchers in the water sector. As the data set used in this study was collected across only 10 months, we propose that future work further validates the clustering methods using longer-period datasets from multiple locations. This study was designed based solely on DWM data. However, the inclusion of socio-demographic data could offer more relevant and valuable insights. To this end, 
further investigation of clustering should be conducted on DWM data with socio-demographic data. In addition, future studies should also focus on feature engineering, as the inclusion of new data should provide more opportunities to create new features. Lastly, future studies can be conducted to understand the characteristics of different groups of consumers in terms of adopting hand hygiene practices.

Funding

This research was funded by the Australian Research Council (ARC), grant number LP160100215, which also includes funding from the following industry partners: Yarra Valley Water, City West Water, Southeast Water, and Aquiba.

\section{References:}

1. The Global Risks Report. 2019, World Economic Forum: Geneva, Switzerland.

2. Behera, B.K., P.K. Rout, and S. Behera, Move Towards Zero Hunger, in Move Towards Zero Hunger. 2019, Springer. p. 1-35.

3. LaVanchy, G.T., M.W. Kerwin, and J.K. Adamson, Beyond 'Day Zero': insights and lessons from Cape Town (South Africa). Hydrogeology Journal, 2019. 27(5): p. 1537-1540.

4. Nguyen, K.A., et al., Water demand forecasting with AUTOFLOWC using State-Space approach, in 8th International Congress on Environmental Modelling and Software. 2016.

5. Liu, A. and P. Mukheibir, Digital metering feedback and changes in water consumption - A review. Resources, Conservation and Recycling, 2018. 134: p. 136-148.

6. Nguyen, K.A., et al., Re-engineering traditional urban water management practices with smart metering and informatics. Environmental Modelling \& Software, 2018. 101: p. 256267.

7. Cominola, A., et al., Data mining to uncover heterogeneous water use behaviors from smart meter data. Water Resources Research, 2019.

8. Saxena, A., et al., A review of clustering techniques and developments. Neurocomputing, 2017. 267: p. 664-681.

9. Rahim, M.S. and T. Ahmed, An initial centroid selection method based on radial and angular coordinates for K-means algorithm, in 2017 20th International Conference of Computer and Information Technology (ICCIT). 2017, IEEE. p. 1-6.

10. Vieira, P., C. Jorge, and D. Covas, Efficiency assessment of household water use. Urban Water Journal, 2018. 15(5): p. 407-417.

11. Garcia, D., et al., Big Data Analytics and Knowledge Discovery Applied to Automatic Meter Readers, in Real-time Monitoring and Operational Control of Drinking-Water Systems. 2017, Springer International Publishing: Cham, Switzerland. p. 401-423.

12. Ji, Y., et al. Analysis of urban residential water consumption based on smart meters and fuzzy clustering. in Proceedings - 15th IEEE International Conference on Computer and Information Technology, CIT 2015, 14th IEEE International Conference on Ubiquitous Computing and Communications, IUCC 2015, 13th IEEE International Conference on Dependable, Autonomic and Secure Computing, DASC 2015 and 13th IEEE International Conference on Pervasive Intelligence and Computing, PICom 2015. 2015.

13. Cheifetz, N., et al., Modeling and clustering water demand patterns from real-world smart meter data. Drinking Water Engineering and Science, 2017. 10(2): p. 75-82.

14. Cominola, A., et al., Profiling residential water users' routines by eigenbehavior modelling. 2016.

15. Cardell-Oliver, R., Water use signature patterns for analyzing household consumption using medium resolution meter data. Water Resources Research, 2013. 49(12): p. 8589-8599.

16. Solanas, J.L. and M.R. Cussó, Multivariate consumption profiling (MCP) for intelligent meter systems: a methodology to define categories and levels. Water Supply, 2010. 10(5): p. 710720. 
17. Padulano, R. and G. Del Giudice, A Mixed Strategy Based on Self-Organizing Map for Water Demand Pattern Profiling of Large-Size Smart Water Grid Data. Water Resources Management, 2018. 32(11): p. 3671-3685.

18. Rahim, M.S., et al. Predicting Household Water Consumption Events: Towards a Personalised Recommender System to Encourage Water-conscious Behaviour. in 2019 International Joint Conference on Neural Networks (IJCNN). 2019.

19. Rahim, M.S., et al., Machine Learning and Data Analytic Techniques in Digital Water Metering: A Review. Water, 2020. 12(1).

20. Rahim, M.S., et al., Advanced household profiling using digital water meters. Journal of Environmental Management, 2021. 288: p. 112377.

21. Rahim, M.S., et al., Digital Water Meters for Advanced Residential Customer Profiling to Promote Water Conservation.

22. Guo, G., J. Zhang, and N. Yorke-Smith, Leveraging multiviews of trust and similarity to enhance clustering-based recommender systems. Knowledge-Based Systems, 2015. 74: p. 1427.

23. Schultz, W., Social comparison as a tool to promote residential water conservation. Frontiers in Water, 2019. 1: p. 2.

24. Han, J., M. Kamber, and J. Pei, 10 - Cluster Analysis: Basic Concepts and Methods, in Data Mining (Third Edition), J. Han, M. Kamber, and J. Pei, Editors. 2012, Morgan Kaufmann: Boston. p. 443-495.

25. Bolorinos, J., N.K. Ajami, and R. Rajagopal, Consumption change detection for urban planning: Monitoring and segmenting water customers during drought. Water Resources Research, 2020. 56(3): p. e2019WR025812.

26. Wang, J., R. Cardell-Oliver, and W. Liu, An incremental algorithm for discovering routine behaviours from smart meter data. Knowledge-Based Systems, 2016. 113: p. 61-74.

27. Nguyen, K.A., R.A. Stewart, and H. Zhang, An autonomous and intelligent expert system for residential water end-use classification. Expert Systems with Applications, 2014. 41(2): p. 342-356.

28. Nguyen, K., et al., Next Generation Machine Learning for Urban Water Management. Water e-Journal, 2020. 5(1): p. 1-7.

29. Stewart, R.A., et al., Web-based knowledge management system: linking smart metering to the future of urban water planning. Australian Planner, 2010. 47(2): p. 66-74.

30. Zheng, A. and A. Casari, Feature engineering for machine learning: principles and techniques for data scientists. 2018: " O'Reilly Media, Inc.".

31. Domingos, P., A few useful things to know about machine learning. Communications of the ACM, 2012. 55(10): p. 78-87.

32. Willis, R.M., et al., Alarming visual display monitors affecting shower end use water and energy conservation in Australian residential households. Resources, Conservation and Recycling, 2010. 54(12): p. 1117-1127.

33. Jorgensen, B.S., et al., Aligning theory and measurement in behavioral models of water conservation. Water Policy, 2015. 17(4): p. 762-776.

34. Makki, A.A., et al., Revealing the determinants of shower water end use consumption: enabling better targeted urban water conservation strategies. Journal of Cleaner Production, 2013. 60: p. 129-146.

35. Willis, R., et al., End use water consumption in households: impact of socio-demographic factors and efficient devices. Journal of Cleaner Production, 2013. 60: p. 107-115.

36. Lam, D. and D.C. Wunsch, Chapter 20 - Clustering, in Academic Press Library in Signal Processing, P.S.R. Diniz, et al., Editors. 2014, Elsevier. p. 1115-1149.

37. MacQueen, J. Some methods for classification and analysis of multivariate observations. in Proceedings of the fifth Berkeley symposium on mathematical statistics and probability. 1967. Oakland, CA, USA. 
38. Dueck, D., Affinity propagation: clustering data by passing messages. 2009: Citeseer.

39. Cheng, Y., Mean shift, mode seeking, and clustering. IEEE transactions on pattern analysis and machine intelligence, 1995. 17(8): p. 790-799.

40. Ng, A.Y., M.I. Jordan, and Y. Weiss, On spectral clustering: Analysis and an algorithm. Advances in neural information processing systems, 2002. 2: p. 849-856.

41. Zhang, T., R. Ramakrishnan, and M. Livny, BIRCH: A new data clustering algorithm and its applications. Data Mining and Knowledge Discovery, 1997. 1(2): p. 141-182.

42. Sasirekha, K. and P. Baby, Agglomerative hierarchical clustering algorithm- $a$. International Journal of Scientific and Research Publications, 2013. 83: p. 83.

43. Jolliffe, I.T. and J. Cadima, Principal component analysis: a review and recent developments. Philosophical Transactions of the Royal Society A: Mathematical, Physical and Engineering Sciences, 2016. 374(2065): p. 20150202.

44. Ringnér, M., What is principal component analysis? Nature Biotechnology, 2008. 26(3): p. 303-304.

45. Clustering. [cited 2021 28/03]; Available from: https://scikitlearn.org/stable/modules/clustering.html.

46. Anderson, B., Pattern Recognition: An introduction. 2019: Scientific e-Resources.

47. Jain, A.K., M.N. Murty, and P.J. Flynn, Data clustering: a review. ACM computing surveys (CSUR), 1999. 31(3): p. 264-323.

48. Nagy, G., State of the art in pattern recognition. Proceedings of the IEEE, 1968. 56(5): p. 836863.

49. Baeza-Yates, R.A., Introduction to Data Structures and Algorithms Related to Information Retrieval. 1992.

50. Minitab. Linkage methods for Cluster Observations. 2019 [cited 2020 15/06/2020]; Linkage methods for Cluster Observations]. Available from: https://support.minitab.com/enus/minitab/18/help-and-how-to/modeling-statistics/multivariate/how-to/clusterobservations/methods-and-formulas/linkage-methods/.

51. Lachev, T. and E. Price, Applied Microsoft Power BI: Bring your data to life! 2018: Prologika Press.

52. Waskom, M., et al., Seaborn: statistical data visualization. URL: https://seaborn. pydata. org/(visited on 2017-05-15), 2014.

53. WHO, W.H.O., Water, sanitation, hygiene and waste management for COVID-19: technical brief, 03 March 2020. 2020, World Health Organization.

54. House, L.W. and J.D. House, Shifting the timing of customer water consumption. JournalAmerican Water Works Association, 2012. 104(2): p. E82-E92.

55. Milligan, G.W. and M.C. Cooper, Methodology review: Clustering methods. Applied psychological measurement, 1987. 11(4): p. 329-354. 


\section{Appendix A. Supplementary materials}

Table S1. Clustering quality analysis for different linkage methods at different intervals

\begin{tabular}{|c|c|c|c|c|c|c|c|c|}
\hline \multirow[t]{2}{*}{ Event } & \multirow{2}{*}{$\begin{array}{c}\text { Type of } \\
\text { day }\end{array}$} & \multirow{2}{*}{$\begin{array}{l}\text { Linkage } \\
\text { method }\end{array}$} & \multicolumn{2}{|c|}{15 minutes interval } & \multicolumn{2}{|c|}{30 minutes interval } & \multicolumn{2}{|c|}{60 minutes interval } \\
\hline & & & $\begin{array}{l}\text { Silhouette } \\
\text { score }\end{array}$ & $\begin{array}{l}\text { Calinski- } \\
\text { Harabaz index } \\
\text { score }\end{array}$ & $\begin{array}{l}\text { Silhouette } \\
\text { score }\end{array}$ & $\begin{array}{l}\text { Calinski-Harabaz } \\
\text { index score }\end{array}$ & $\begin{array}{l}\text { Silhouette } \\
\text { score }\end{array}$ & $\begin{array}{l}\text { Calinski-Harabaz } \\
\text { index score }\end{array}$ \\
\hline \multirow[t]{8}{*}{ Shower } & \multirow{4}{*}{ Weekday } & average & 0.448506853 & 8.527780474 & 0.389057434 & 5.959777119 & 0.308926746 & 9.180718971 \\
\hline & & complete & 0.372250317 & 7.911656809 & 0.122297591 & 15.51640278 & 0.239199749 & 17.06467639 \\
\hline & & single & 0.427971072 & 5.783713894 & 0.363332933 & 4.466860595 & 0.316601415 & 6.925762188 \\
\hline & & ward & 0.064585126 & 16.48461465 & 0.085704233 & 23.35527664 & 0.131193544 & 38.18387255 \\
\hline & \multirow{4}{*}{ Weekend } & average & 0.387995798 & 4.969430565 & 0.350154662 & 4.66748129 & 0.257707829 & 6.091642695 \\
\hline & & complete & 0.279966194 & 6.599699951 & 0.169437378 & 7.613812463 & 0.146911062 & 14.73137115 \\
\hline & & single & 0.368262744 & 4.756962776 & 0.314006442 & 4.490504089 & 0.194980232 & 2.840232588 \\
\hline & & ward & 0.054635753 & 10.45767908 & 0.05713935 & 17.53167742 & 0.108168696 & 29.74925878 \\
\hline \multirow[t]{8}{*}{ Bathtub } & \multirow{4}{*}{ Weekday } & average & 0.400183455 & 6.846952511 & 0.373110812 & 10.51186476 & 0.348201581 & 14.25684781 \\
\hline & & complete & 0.312699229 & 8.869961496 & 0.304695483 & 16.15385684 & 0.370836873 & 22.35718599 \\
\hline & & single & 0.367654212 & 3.758340884 & 0.327866855 & 5.689559169 & 0.258029231 & 4.217600053 \\
\hline & & ward & 0.295876159 & 12.46834084 & 0.20133816 & 18.6062978 & 0.200069113 & $\mathbf{3 4 . 6 7 3 3 5 9 4 2}$ \\
\hline & \multirow{4}{*}{ Weekend } & average & 0.461686018 & 9.093022361 & 0.438843043 & 10.83575144 & 0.414427679 & 21.86735394 \\
\hline & & complete & 0.395908944 & 9.271544696 & 0.386051994 & 17.86323387 & 0.367811647 & 35.01426593 \\
\hline & & single & 0.401628429 & 4.850188956 & 0.395706999 & 3.740394846 & 0.276570993 & 3.006134119 \\
\hline & & ward & 0.355189864 & 16.13754607 & 0.297756946 & 23.48946723 & 0.383651228 & 42.70918856 \\
\hline \multirow{8}{*}{$\begin{array}{l}\text { Washing } \\
\text { machine }\end{array}$} & \multirow{4}{*}{ Weekday } & average & 0.283630271 & 5.064187824 & 0.281818241 & 5.817987705 & 0.288801034 & 10.27674789 \\
\hline & & complete & 0.29916421 & 7.429594973 & 0.120457566 & 10.90812392 & 0.134126337 & 22.390375 \\
\hline & & single & 0.238873969 & 2.720056885 & 0.239247221 & 3.989578345 & 0.175324957 & 3.37178633 \\
\hline & & ward & 0.111866528 & 9.842611968 & 0.097792561 & 17.79484028 & 0.154892491 & 37.58881767 \\
\hline & \multirow{4}{*}{ Weekend } & average & 0.260427015 & 3.210386585 & 0.23152945 & 6.461625555 & 0.214952245 & 6.408004666 \\
\hline & & complete & 0.123262971 & 4.77034938 & 0.174960716 & 14.50455964 & 0.17552722 & 33.89088256 \\
\hline & & single & 0.247076743 & 2.702268244 & 0.199163182 & 3.059571985 & 0.119874696 & 2.78488543 \\
\hline & & ward & 0.124873229 & 11.97837429 & 0.165163019 & 22.23208569 & 0.191602706 & 43.52112063 \\
\hline \multirow[t]{8}{*}{ Dishwasher } & \multirow{4}{*}{ Weekday } & average & 0.483852879 & 6.373322196 & 0.431564782 & 6.462600815 & 0.40715529 & 12.21027227 \\
\hline & & complete & 0.40210014 & 9.269771751 & 0.371042259 & 16.13988977 & 0.357417046 & 19.70524038 \\
\hline & & single & 0.469878616 & 4.885980105 & 0.380708779 & 3.998327193 & 0.375620594 & 4.019743989 \\
\hline & & ward & 0.233929965 & 13.20546727 & 0.27033453 & 21.9835683 & 0.326593531 & 36.57076642 \\
\hline & \multirow{4}{*}{ Weekend } & average & 0.45981917 & 5.360905564 & 0.45143523 & 9.36519768 & 0.41383852 & 9.280031376 \\
\hline & & complete & 0.390831996 & 8.243764828 & 0.40428604 & 10.2314595 & 0.375240228 & 13.80016539 \\
\hline & & single & 0.447383489 & 4.144558588 & 0.410585569 & 5.613689998 & 0.318238961 & 4.497633016 \\
\hline & & ward & 0.395046889 & 11.10108041 & 0.292073135 & 15.70810369 & 0.326733657 & 25.72856855 \\
\hline \multirow[t]{8}{*}{ Irrigation } & \multirow{4}{*}{ Weekday } & average & 0.423492042 & 5.902786329 & 0.455812809 & 10.23541785 & 0.393684221 & 17.54319606 \\
\hline & & complete & 0.281541077 & 9.600119056 & 0.432439422 & 13.19444842 & 0.374403278 & 17.73854126 \\
\hline & & single & 0.386334541 & 5.577191876 & 0.377082886 & 8.177564031 & 0.206190944 & 4.65424353 \\
\hline & & ward & 0.237771667 & 10.14647307 & 0.291315195 & 15.8600189 & 0.208685034 & 24.22976972 \\
\hline & \multirow{4}{*}{ Weekend } & average & 0.400884363 & 4.061446995 & 0.404678698 & 7.747732313 & 0.383231008 & 16.97523022 \\
\hline & & complete & 0.288535336 & 6.555188651 & 0.173686917 & 13.3046751 & 0.32607151 & 18.24875331 \\
\hline & & single & 0.394944274 & 4.373974615 & 0.349175947 & 4.308400813 & 0.206098349 & 2.74666702 \\
\hline & & ward & 0.241033094 & 9.71990254 & 0.230537101 & 16.01134383 & 0.251962115 & 28.24239568 \\
\hline
\end{tabular}




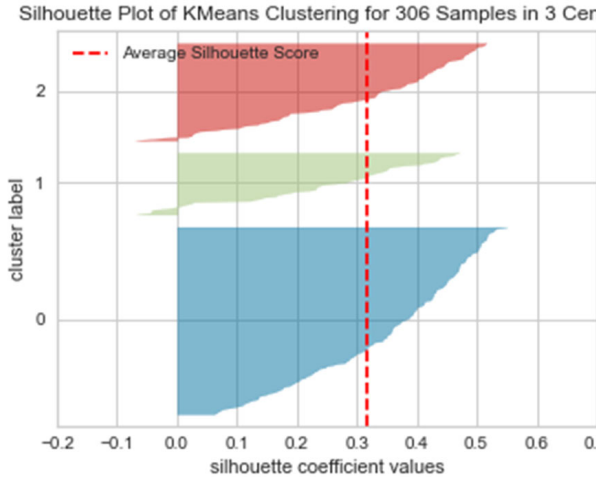

(a)

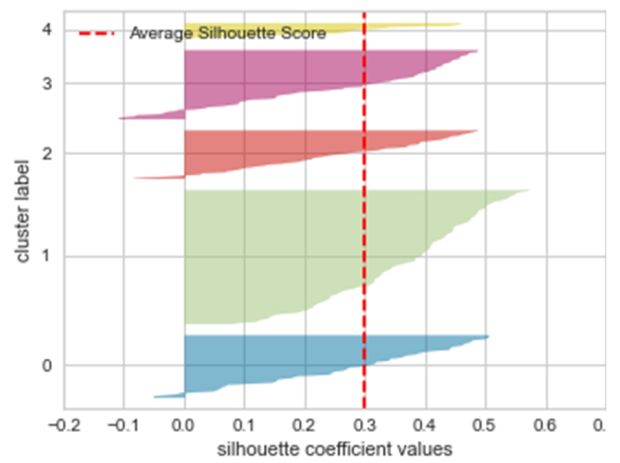

(c)

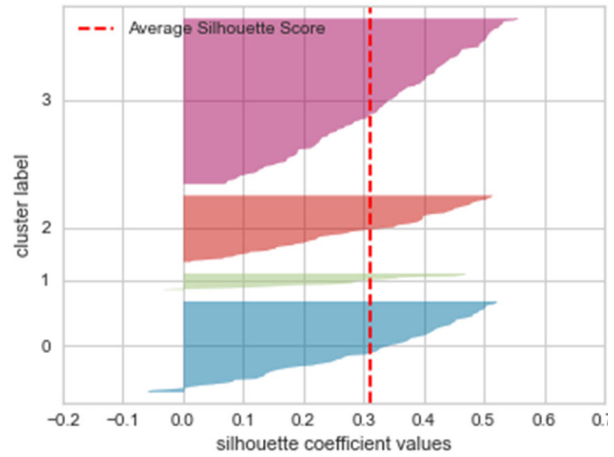

(b)

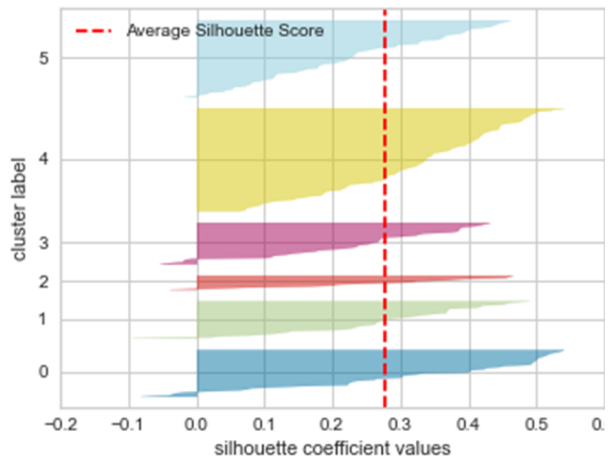

(d)

Figure S2. Silhouette plot of k-means clustering for 306 households in (a) 3, (b) 4, (c) 5, and (d) 6 clusters. 
- Discussed a detailed methodology to analyse water consumption data for clustering.

- Performed a comprehensive experimental study on the data and clustering methods

- Identified the most suitable profiling interval in terms of similarity among the items in each cluster.

- Observed how tap-water usage can determine hand-hygiene practices during COVID-19 like scenarios. 
Md Shamsur Rahim: Conceptualization, Methodology, Data Curation, Software, Writing- Original draft preparation, Visualization. Khoi Anh Nguyen: Conceptualization, Methodology, Data Curation, Writing - Review \& Editing, Funding acquisition. Rodney Anthony Stewart: Conceptualization, Methodology, Writing - Review \& Editing, Funding acquisition. Tanvir Ahmed: Conceptualization, Methodology, Writing - Review \& Editing. Damien Giurco: Conceptualization, Methodology, Writing Review \& Editing, Funding acquisition. Michael Blumenstein: Conceptualization, Methodology, Writing - Review \& Editing, Funding acquisition. 